\title{
Moment asymptotics for parabolic Anderson equation with fractional time-space noise: In Skorokhod regime
}

\author{
Xia Chen ${ }^{1}$ \\ Department of Mathematics, University of Tennessee, Knoxville TN 37996, USA. E-mail: xchen@math.utk.edu
}

Received 8 August 2015; revised 27 December 2015; accepted 28 December 2015

\begin{abstract}
In this paper, we consider the parabolic Anderson equation that is driven by a Gaussian noise fractional in time and white or fractional in space, and is solved in a mild sense defined by Skorokhod integral. Our objective is the precise moment Lyapunov exponent and high moment asymptotics. As far as the long term asymptotics are concerned, some feature given in our theorems is different from what have been observed in the Stratonovich-regime and in the setting of the white time noise. While the difference disappears when it comes to the high moment asymptotics. To achieve our goal, we introduce a variational inequality and use some newly developed tools such as time-space LDP of Feynman-Kac type, linearization by tangent approximation, together with some techniques developed along the line of probability in Banach spaces.
\end{abstract}

Résumé. Nous considérons l'équation d'Anderson parabolique conduite par un bruit gaussien, fractionnaire en temps, et blanc ou fractionnaire en espace, qu'on résout dans un sens faible défini par une intégrale de Skorokhod. Notre objectif est de donner l'exposant de Lyapounov pour les moments, et les asymptotiques des grands moments. Pour les asymptotiques en temps long, nos résultats mettent en évidence des phénomènes différents de ceux observés pour le régime Stratonovich, et dans le cas d'un bruit blanc en temps. Ces différences s'effacent néanmoins lorsque l'on considère les asymptotiques des grands moments. Nos résultats sont obtenus en introduisant une nouvelle inégalité variationnelle, et à l'aide d'outils nouveaux tels qu'un principe de grandes déviations de type Feynman-Kac, la linéarisation par des approximations tangentes, et des techniques inspirées des probabilités dans les espaces de Banach.

MSC: 60F10; 60H15; 60H40; 60J65; 81U10

Keywords: Lyapunov exponent; High moment asymptotics; White and fractional noise; Brownian motion; Parabolic Anderson equation; Feynman-Kac's representation

\section{Introduction}

The parabolic Anderson equation considered in this paper takes form

$$
\left\{\begin{array}{l}
\frac{\partial u}{\partial t}(t, x)=\frac{1}{2} \Delta u(t, x)+V(t, x) \circ u(t, x), \\
u(0, x)=u_{0}(x)
\end{array}\right.
$$

where $V(t, x)$ is a generalized Gaussian field with the covariance function

$$
\operatorname{Cov}(V(t, x), V(s, y))=\gamma_{0}(t-s) \gamma(x-y) \quad(t, x),(s, y) \in \mathbb{R}^{+} \times \mathbb{R}^{d}
$$

\footnotetext{
${ }^{1}$ Research partially supported by the Simons Foundation \#244767.
} 
that is to be further specified in sequel, and "o" represents the Wick product. In a rigorous definition (Definition 3.1, [7]) $u(t, x)$ is given as the mild solution of

$$
u(t, x)=p_{t} * u_{0}(x)+\int_{0}^{t} \int_{\mathbb{R}^{d}} p_{t-s}(x-y) u(s, y) \delta V(s, y),
$$

where the the differential notation " $\delta V(s, x)$ " is used for the Skorokhod integral. Throughout, we assume that

$$
0<\inf _{x \mathbb{R}^{d}} u_{0}(x) \leq \sup _{x \in \mathbb{R}^{d}} u_{0}(x)<\infty .
$$

In a recent work [7], it is proved that when $\gamma_{0}(\cdot)$ is locally integrable and $\gamma(\cdot) \geq 0$ this system yields an unique solution $u(t, x)$ with $\mathbb{E} u(t, x)^{n}<\infty$ for all $n \geq 1$ under the Dalang's condition

$$
\int_{\mathbb{R}^{d}} \frac{1}{1+|\xi|^{2}} \mu(d \xi)<\infty
$$

where $\mu(d \xi)$ is the spectral measure of the non-negative definite function $\gamma(\cdot)$.

The objective of this paper is to investigate the asymptotics

$$
\log \mathbb{E} u(t, x)^{n} \quad \text { as } t \rightarrow \infty \text { or as } n \rightarrow \infty .
$$

The limit related to the long-term asymptotics is known as the moment Lyapunov exponent in literature and the problem is closely related to the issue of intermittency. See [1] for the general information on intermittency. The high moment asymptotics, on the other hand, is essential for understanding the large scale behaviors of the model in the space variable $x$ (see, e.g., [4]). Here we are concerned with the setting where the Gaussian noise is fractional in time and white or fractional in space. More precisely, in our main theorems below,

$$
\left\{\begin{array}{l}
\gamma_{0}(u)=|u|^{-\alpha_{0}} \quad\left(0 \leq \alpha_{0}<1\right) \\
\gamma(\cdot)=\int_{\mathbb{R}^{d}} K(y-\cdot) K(y) d y \text { for some function } K(\cdot) \geq 0 \text { on } \mathbb{R}^{d} \\
\text { there is a } 0<\alpha<2 \text { such that } \gamma(c x)=c^{-\alpha} \gamma(x) \text { for } c>0, x \in \mathbb{R}^{d} .
\end{array}\right.
$$

We point out that the Dalang's condition is satisfied under (1.6). Indeed, the scaling property of $\gamma(\cdot)$ leads to

$$
\mu(d(C \xi))=C^{\alpha} \mu(d \xi) \quad \forall C>0 .
$$

Consequently, for any integer $k \geq 1$

$$
\mu\left\{|\xi| \leq 2^{k}\right\}=2^{\alpha k} \mu\{|\xi| \leq 1\} .
$$

Since $\mu$ is tempered (in particular, $\mu$ is locally finite), $\mu\{|\xi| \leq 1\}<\infty$. Therefore,

$$
\begin{aligned}
\int_{\mathbb{R}^{d}} \frac{1}{1+|\xi|^{2}} \mu(d \xi) & \leq \mu\{|\xi| \leq 1\}+\sum_{k=1}^{\infty} 2^{-2(k-1)} \mu\left\{2^{k-1}<|\xi| \leq 2^{k}\right\} \\
& \leq \mu\{|\xi| \leq 1\}\left\{1+\sum_{k=1}^{\infty} 2^{-2(k-1)} 2^{\alpha k}\right\}<\infty .
\end{aligned}
$$

Here we list some special cases of (1.6).

1. $\alpha_{0}=2-2 H_{0}$ with $1 / 2<H_{0}<1$ and

$$
\gamma(x)=c_{H} \prod_{i=1}^{d}\left|x_{i}\right|^{-\left(2-2 H_{i}\right)} \quad x=\left(x_{1}, \ldots, x_{d}\right) \in \mathbb{R}^{d}
$$


for a suitable constant $c_{H}>0$, where $1 / 2<H_{i}<1(1 \leq i \leq d)$ satisfying $H_{1}+\cdots+H_{d}>d-1$. In this case $V(t, x)$ represents a fractional Gaussian noise formally given as

$$
V(t, x)=\frac{\partial^{d+1} W^{H}}{\partial t \partial x_{1} \cdots \partial x_{d}}\left(t, x_{1}, \ldots, x_{d}\right),
$$

where $W^{H}(t, x)\left((t, x) \in \mathbb{R}^{+} \times \mathbb{R}^{d}\right)$ is a fractional Brownian sheet with the Hurst parameter $H=\left(H_{0}, H_{1}, \ldots, H_{d}\right)$. In accordance with the conditions given in (1.6), $K(x)$ is a constant multiple of the function

$$
\prod_{j=1}^{d}\left|x_{j}\right|^{-\frac{3-2 H_{j}}{2}} \quad x=\left(x_{1}, \ldots, x_{d}\right) \in \mathbb{R}^{d}
$$

and $\alpha=2 d-2\left(H_{1}+\cdots+H_{d}\right)$ and $K(x)$.

2. $\alpha_{0}=2-2 H_{0}$ with $1 / 2<H_{0}<1, d=1=c \delta_{0}(x)$ where $c>0$ is a suitable constant and $\delta_{0}(\cdot)$ represents the Dirac function on $\mathbb{R}$. In this case, $V(t, x)$ represents the noise

$$
V(t, x)=\frac{\partial^{2} W^{\left(H_{0}, 1 / 2\right)}}{\partial t \partial x}(t, x)
$$

that is colored in time and white in space. In accordance with (1.6), $\alpha=1$ and $K(\cdot)$ appears as a constant multiple of the Dirac function on $\mathbb{R}$.

3. In light of the physical laws such as Newton's gravitation law and Coulomb's electrostatics law, it makes sense to consider the case when

$$
\gamma(x)=C|x|^{-\alpha} \quad x \in \mathbb{R}^{d},
$$

where $K(x)$ is a suitable constant multiple of the function

$$
|x|^{-\frac{d+\alpha}{2}} \quad x \in \mathbb{R}^{d} .
$$

In addition, the setting $\alpha_{0}=0$ corresponds to a time-independent noise $V(x)$.

Comparison is part of the reasons that motivate this work. It includes the comparison between the Gaussian noises white and colored in time, and the comparison between the Skorokhod-regime and Stratonovich-regime. In the case when $d=1$ and when both $\gamma_{0}(\cdot)$ and $\gamma(\cdot)$ are Dirac function on $\mathbb{R}$ (the case is known as "white in time and white in space"), it was recently confirmed ([3]) that

$$
\lim _{t \rightarrow \infty} \frac{1}{t} \log \mathbb{E} u(t, x)^{n}=\frac{1}{24} n\left(n^{2}-1\right) \quad n=1,2, \ldots
$$

Another existing piece of work is when the Wick product "o" in (1.1) is replaced by the usual product or, the Skorokhod integral in (1.2) is replaced by the corresponding Stratonovich integral, where the solution has the FeynmanKac representation ([9])

$$
\tilde{u}(t, x)=\mathbb{E}_{x}\left[\exp \left\{\int_{0}^{t} V(t-s, B(s)) d s\right\} \tilde{u}_{0}(B(t))\right]
$$

under the more restrictive condition $\alpha+2 \alpha_{0}<2$, where $B(s)$ is a $d$-dimensional Brownian motion independent of $V(t, x)$ and the notation " $\mathbb{E}_{x}$ " is for the expectation with respect to the Brownian motion $B(s)$ starting at $x$. It is shown (Theorem 6.1, [5]) that

$$
\lim _{t \rightarrow \infty} t^{-\frac{4-\alpha-2 \alpha_{0}}{2-\alpha}} \log \mathbb{E} \tilde{u}^{n}(t, x)=\left(\frac{1}{2}\right)^{\frac{2}{2-\alpha}} n^{\frac{4-\alpha}{2-\alpha}} \mathcal{E} \quad n=1,2, \ldots,
$$


where $\mathcal{E}$ is given by the following variational characterization

$$
\mathcal{E}=\sup _{g \in \mathcal{A}_{d}}\left\{\int_{0}^{1} \int_{0}^{1} \int_{\mathbb{R}^{d} \times \mathbb{R}^{d}} \frac{\gamma(x-y)}{|s-r|^{\alpha_{0}}} g^{2}(s, x) g^{2}(r, y) d x d y d r d s-\frac{1}{2} \int_{0}^{1} \int_{\mathbb{R}^{d}}\left|\nabla_{x} g(s, x)\right|^{2} x d s\right\}
$$

and $\mathcal{A}_{d}$ is the function class defined as

$$
\mathcal{A}_{d}=\left\{g ; g(s, \cdot) \in W^{1,2}\left(\mathbb{R}^{d}\right) \text { and } \int_{\mathbb{R}^{d}} g^{2}(s, x) d x=1 \forall 0 \leq s \leq 1\right\} .
$$

The finiteness of $\mathcal{E}$ and its relation to other variational forms are established in Lemma 7.2 and Lemma 7.4 in [5] under the extra condition $\alpha+2 \alpha_{0}<2$. In Lemma 5.1 below, the finiteness of $\mathcal{E}$ is re-established under a very general setting that takes (1.6) as a special case.

In comparison with (1.9), $u(t, x)$ in our setting can be formally (see (2.3) below for the mathematical construction) represented in terms of the Wick exponential moment

$$
u(t, x)=\mathbb{E}_{x}\left[\exp \left\{\int_{0}^{t} V(t-s, B(s)) d s-\frac{1}{2} \int_{0}^{t} \int_{0}^{t} \frac{\gamma(B(s)-B(r))}{|s-r|^{\alpha_{0}}} d r d s\right\} u_{0}(B(t))\right] .
$$

As far as the moment asymptotics are concerned, a natural problem is to understand the possible impact by adding the correction term (i.e., the random Hamiltonian appearing in (1.13)), or by switching from the Gaussian noise that is white in time to the one that is fractional in time.

Theorem 1.1. Under the assumption (1.6),

$$
\lim _{t \rightarrow \infty} t^{-\frac{4-\alpha-2 \alpha_{0}}{2-\alpha}} \log \mathbb{E} u^{n}(t, x)=n\left(\frac{n-1}{2}\right)^{\frac{2}{2-\alpha}} \mathcal{E} \quad n=1,2, \ldots
$$

for every $x \in \mathbb{R}^{d}$, where $\mathcal{E}$ is defined by the variation in (1.11).

Comparison among (1.8), (1.10) and (1.14) shows that in all three settings, namely the white-time (where $\alpha_{0}=1$ ) regime, the Stratonovich regime, and the Skorokhod regime with fractional-time, the asymptotic rate is given in a consistent way. On the other hand, the right-hand sides of (1.8), (1.10) and (1.14) appear to be three distinct functions in $n$.

A special case is when $\alpha_{0}=0$ (i.e, $\gamma_{0}(u)=1$ ), where the Gaussian noise in the equation (1.1) is time-independent (i.e., in the form of $V(x)$ ) and where the representation (1.13) is mathematically rigorous as the time integral and the random Hamiltonian in (1.13) are properly defined (Section A.1, [2]). In this case we have (Lemma 7.5, [4]) that

$$
\mathcal{E}=\sup _{g \in \mathcal{F}_{d}}\left\{\int_{\mathbb{R}^{d} \times \mathbb{R}^{d}} \gamma(x-y) g^{2}(x) g^{2}(y) d x d y-\frac{1}{2} \int_{\mathbb{R}^{d}}|\nabla g(x)|^{2} d x\right\},
$$

where

$$
\mathcal{F}_{d}=\left\{g \in W^{1,2}\left(\mathbb{R}^{d}\right) ; \int_{\mathbb{R}^{d}}|g(x)|^{2} d x=1\right\} ;
$$

and that

$$
\lim _{t \rightarrow \infty} t^{-\frac{4-\alpha}{2-\alpha}} \log \mathbb{E} u^{n}(t, x)=n\left(\frac{n-1}{2}\right)^{\frac{2}{2-\alpha}} \mathcal{E} \quad n=1,2, \ldots,
$$

provided that the space covariance $\gamma(\cdot)$ satisfies (1.6). 
As suggested by (1.8), the case when $V(t, x)$ is white in time, i.e., $\gamma_{0}(u)=\delta_{0}$ (or, $\alpha_{0}=1$ ) is not covered by Theorem 1.1. On the other hand, the computation of asymptotic Lyapunov moment is not hard in this setting. Indeed, by Feynman-Kac moment representation (Theorem 5.3, [8])

$$
\mathbb{E} u^{n}(t, x)=\mathbb{E}_{0}\left[\exp \left\{\frac{1}{2} \int_{0}^{t} \sum_{j \neq k}^{n} \gamma\left(B_{j}(s)-B_{k}(s)\right) d s\right\} \prod_{j=1}^{n} u_{0}\left(B_{j}(t)\right)\right] .
$$

A standard argument by semi-group method gives that

$$
\begin{aligned}
& \lim _{t \rightarrow \infty} \frac{1}{t} \log \mathbb{E} u^{n}(t, x) \\
& =\left(\frac{1}{2}\right)^{\frac{2}{2-\alpha}} \sup _{g \in \mathcal{F}_{n d}}\left\{\sum_{j \neq k}^{n} \int_{\left(\mathbb{R}^{d}\right)^{n}} \gamma\left(x_{j}-x_{k}\right) g^{2}(x) d x-\frac{1}{2} \int_{\left(\mathbb{R}^{d}\right)^{n}}|\nabla g(x)|^{2} d x\right\},
\end{aligned}
$$

where $x=\left(x_{1}, \ldots, x_{n}\right) \in\left(\mathbb{R}^{d}\right)^{n}$ and $\mathcal{F}_{n d}$ is defined by (1.16) with $d$ being replaced by $n d$.

The story does not end here. The right-hand side of (1.18), denoted by $\lambda_{n}$, does not show explicitly how it depends on $n$. In particular, it does not tell whether or not the intermittency criteria " $n^{-1} \lambda_{n}<(n+1)^{-1} \lambda_{n+1}(n=1,2, \ldots)$ " holds. In light of Theorem 1.1, we wonder if the limit in (1.18) can be formulated in terms of the variation $\mathcal{E}$. On the other hand, we claim that (1.15) holds here. Indeed, replacing $|\cdot|^{-\alpha_{0}}$ by $\delta_{0}(\cdot)$ in (1.11),

$$
\begin{aligned}
\mathcal{E} & =\sup _{g \in \mathcal{A}_{d}}\left\{\int_{0}^{1} \int_{\mathbb{R}^{d} \times \mathbb{R}^{d}} \gamma(x-y) g^{2}(s, x) g^{2}(s, y) d x d y d s-\frac{1}{2} \int_{0}^{1} \int_{\mathbb{R}^{d}}\left|\nabla_{x} g(s, x)\right|^{2} d x d s\right\} \\
& \leq \int_{0}^{1} \sup _{g \in \mathcal{A}_{d}}\left\{\int_{\mathbb{R}^{d} \times \mathbb{R}^{d}} \gamma(x-y) g^{2}(s, x) g^{2}(s, y) d x d y-\frac{1}{2} \int_{\mathbb{R}^{d}}\left|\nabla_{x} g(s, x)\right|^{2} d x\right\} d s \\
& =\sup _{g \in \mathcal{F}_{d}}\left\{\int_{\mathbb{R}^{d} \times \mathbb{R}^{d}} \gamma(x-y) g^{2}(x) g^{2}(y) d x d y-\frac{1}{2} \int_{\mathbb{R}^{d}}|\nabla g(x)|^{2} d x\right\} .
\end{aligned}
$$

The opposite estimate is automatic, as $\mathcal{F}_{d}$ can be viewed as a subclass of $\mathcal{A}_{d}$.

This gives another reason for pursuing this link: In the study of bosonic quantum system (see [10] for the general information), an important problem is the relation between the variation appearing on the right-hand side of (1.18) and the variation $\mathcal{E}$ in (1.15). The former represents the ground state energy of the $n$-body problem and the latter is known as Hartree's energy. Hartree's theory formulates an asymptotic relation between them as $n \rightarrow \infty-$ we refer an interested reader to [11] for the latest development in this area. The concerned here is about the relation for each fixed integer $n \geq 1$. Given (1.18), it is to ask, in probability language, for writing the asymptotic Lyapunov moment in terms of $\mathcal{E}$ in the case when $V(t, x)$ is white in time. More specifically, we conjecture that

$$
\lim _{t \rightarrow \infty} \frac{1}{t} \log \mathbb{E} u^{n}(t, x)=\left(\frac{1}{2}\right)^{\frac{2}{2-\alpha}} n\left(n^{\frac{2}{2-\alpha}}-1\right) \mathcal{E} \quad n=1,2, \ldots
$$

To the author's best knowledge, the conjecture is confirmed only when $d=1, \gamma_{0}=\gamma=\delta_{0}$ where $\mathcal{E}=1 / 6((1.27)$, [4]) and (1.19) becomes (1.8).

As for the high moment asymptotics, we have

Theorem 1.2. Under the assumption (1.6)

$$
\lim _{n \rightarrow \infty} n^{-\frac{4-\alpha}{2-\alpha}} \log \mathbb{E} u^{n}(t, x)=\left(\frac{1}{2}\right)^{\frac{2}{2-\alpha}} t^{\frac{4-\alpha-2 \alpha_{0}}{2-\alpha}} \mathcal{E}
$$

for every $t>0$ and $x \in \mathbb{R}^{d}$, where $\mathcal{E}$ is defined by the variation in (1.11). 
The limit form given in Theorem 1.2 is exactly the same as the one achieved (Proposition 3.1, [4]) in Stratonovichregime, except that the condition has been weaken from " $\alpha+2 \alpha_{0}<2$ " to " $\alpha<2$ " thank to the correction term in (1.13). In addition, the constant appearing in (1.20) is in consistence to what we have seen in the setting of "white in time" (Proposition 3.3, [4]). This observation suggests that unlike in the context of long term asymptotics, the correction term in (1.13) does not play a role in deciding the form of the high moment asymptotics.

Perhaps it is worth mentioning that the study of the moment asymptotics in Skorokhod regime is relatively underdeveloped, especially at the level of precision given in our theorems. In comparison to the Feynman-Kac formula in (1.9), the the extra correction term in (1.13) indicates a major challenge in our setting. In addition, the time integral and the random Hamiltonian on the right-hand side of (1.13) are not always properly defined under our assumption. In dealing with these difficulties, we introduce a variational inequality that linearizes our model in the proof of the lower bounds. The argument for the upper bounds relies on the compactfication procedure to which the large deviation theory and some techniques developed along the line of probability in Banach space play some essential roles. Finally, a newly developed Feynman-Kac type of large deviation method (Proposition 3.1, [5]) is critical for capturing the precise variation in Theorem 1.1 and Theorem 1.2.

Feynman-Kac's representation involves a $d$-dimensional Brownian motion $B(s)$ independent of the Gaussian noise $W$. In the rest of the paper, we use " $\mathbb{E}$ " and " $\mathbb{E}_{x}$ " for the expectation with respect to, respectively, $V$ and $B(s)$ as $B(0)=x$. In some places, " $\mathbb{E}_{x}$ " is also used for the expectation with respect to several independent Brownian motions starting at the same point $x$.

\section{A variational inequality}

For possible future reference, we consider a more general setting in this section: Let $V(t, x)$ be a generalized meanzero Gaussian field on $\mathbb{R}^{d}$ with the covariance structure described in (1.2). More precisely, $V$ is defined as a random linear functional on $\mathcal{S}\left(\mathbb{R}^{d+1}\right)$, the Schwartz space of infinitely smooth and rapidly decreasing functions on $\mathbb{R}^{d+1}$, such that $\langle V, \varphi\rangle\left(\varphi \in \mathcal{S}\left(\mathbb{R}^{d+1}\right)\right)$ is a mean zero Gaussian field with

$$
\begin{aligned}
& \operatorname{Cov}(\langle V, \varphi\rangle,\langle V, \psi\rangle) \\
& \quad=\int_{\mathbb{R}^{d+1} \times \mathbb{R}^{d+1}} \gamma_{0}(u-v) \gamma(x-y) \varphi(u, x) \psi(v, y) d u d v d x d y \quad \varphi, \psi \in \mathcal{S}\left(\mathbb{R}^{d+1}\right) .
\end{aligned}
$$

By Bochner representation, there are tempered, positive and symmetric measures $\mu_{0}(d \lambda)$ on $\mathbb{R}$ and $\mu(d \xi)$ on $\mathbb{R}^{d}$ known as the spectral measures, such that

$$
\gamma_{0}(u)=\int_{\mathbb{R}} e^{i \lambda u} \mu_{0}(d \lambda) \quad \text { and } \quad \gamma(x)=\int_{\mathbb{R}^{d}} e^{i \xi \cdot x} \mu(d \xi) \quad u \in \mathbb{R}, x \in \mathbb{R}^{d} .
$$

Thus, (2.1) can be rewritten as

$$
\operatorname{Cov}(\langle V, \varphi\rangle,\langle V, \psi\rangle)=\int_{\mathbb{R}^{d+1}} \mathcal{F}(\varphi)(\lambda, \xi) \overline{\mathcal{F}(\psi)(\lambda, \xi)} \mu_{0}(d \lambda) \mu(d \xi)
$$

where $\mathcal{F}(\varphi)$ represents the Fourier transform of $\varphi$ :

$$
\mathcal{F}(\varphi)(\lambda, \xi)=\int_{\mathbb{R}^{d+1}} e^{i \lambda u+i \xi \cdot x} \varphi(u, x) d u d x
$$

Throughout this section, $\gamma_{0}(\cdot)$ is locally integrable and $\gamma(\cdot)$ is non-negative and satisfies the Dalang's condition (1.5).

For any $\varepsilon>0$, let $p_{\varepsilon}(u, x)$ be the density function of the centered normal distribution on $\mathbb{R}^{d+1}$ with covariance matrix $\varepsilon I_{d+1}$, where $I_{d+1}$ is the $(d+1) \times(d+1)$ identical matrix. Set

$$
V_{\varepsilon}(u, x)=\left\langle V, p_{\varepsilon}(\cdot-u, \cdot-x)\right\rangle .
$$


Then $V_{\varepsilon}(u, x)$ is a point-wise defined Gaussian field on $\mathbb{R}^{d+1}$. Under this construction

$$
\int_{0}^{t} V_{\varepsilon}\left(t-s, B_{s}\right) d s=\left\langle V, \psi_{t}\right\rangle
$$

with

$$
\psi_{t}(u, x)=\int_{0}^{t} p_{\varepsilon}(u-(t-s), x-B(s)) d s .
$$

Further, the random field

$$
u_{\varepsilon}(t, x)=\mathbb{E}_{x}\left[\exp \left\{\left\langle V, \psi_{t}\right\rangle-\frac{1}{2} \int_{\mathbb{R}^{d+1}}\left|\mathcal{F}\left(\psi_{t}\right)(\lambda, \xi)\right|^{2} \mu_{0}(d \lambda) \mu(d \xi)\right\} u_{0}(B(t))\right]
$$

solves the heat equation

$$
\left\{\begin{array}{l}
\frac{\partial u}{\partial t}(t, x)=\frac{1}{2} \Delta u(t, x)+V_{\varepsilon}(t, x) \circ u(t, x), \\
u(0, x)=u_{0}(x) .
\end{array}\right.
$$

Finally, under the Dalang's condition (1.5) the limit

$$
u(t, x) \equiv \lim _{\varepsilon \rightarrow 0^{+}} u_{\varepsilon}(t, x)-\mathcal{L}^{p}(\Omega)
$$

exists for every $(t, x) \in \mathbb{R}^{+} \times \mathbb{R}^{d}$ and $p>1$ and the limiting field $u(t, x)$ solves the equation (1.1) (Theorem 3.6, [7]).

From the Feynman-Kac representation given in (2.3), one can see that $u_{\varepsilon}(t, x)$ is monotonic in the initial condition $u_{0}(\cdot)$. The monotonicity passes to $u(t, x)$ through the limiting procedure. In view of the assumption $(1.5), u(t, x) \geq 0$ as (1.1) has zero solution under the zero-initial condition. Further, let $\hat{u}(t, x)$ be the solution of $(1.1)$ with $u_{0}(x) \equiv 1$. By linearity $\underline{u}(t, x)=\left\{\inf _{y \in \mathbb{R}^{d}} u_{0}(y)\right\} \hat{u}(t, x)$ and $\bar{u}(t, x)=\left\{\sup _{y \in \mathbb{R}^{d}} u_{0}(y)\right\} \hat{u}(t, x)$ solve $(1.1)$ with the initial conditions $\underline{u}_{0}(x)=\inf _{y \in \mathbb{R}^{d}} u_{0}(y)$ and $\bar{u}_{0}(x)=\sup _{y \in \mathbb{R}^{d}} u_{0}(y)$, respectively. Thus, the monotonicity in initial data implies

$$
\left\{\inf _{y \in \mathbb{R}^{d}} u_{0}(y)\right\} \hat{u}(t, x) \leq u(t, x) \leq\left\{\sup _{y \in \mathbb{R}^{d}} u_{0}(y)\right\} \hat{u}(t, x) \quad \text { a.s. }
$$

To prove Theorem 1.1 and Theorem 1.2, therefore, we need only to consider the case when $u_{0}(x)=1$. For this reason, " $u_{0}(x)=1$ " is assumed in the rest of the paper.

Proposition 2.1. Let $\gamma_{0}(\cdot)$ be locally integrable and let $\gamma(\cdot) \geq 0$ and satisfy the Dalang's condition (1.5). For any $p>1, t>0$ and $x \in \mathbb{R}^{d}$

$$
\begin{aligned}
& \left\{\mathbb{E} u(t, x)^{p}\right\}^{1 / p} \\
& \geq \sup _{h \in \mathcal{H}_{V}} \mathbb{E}_{0} \exp \left\{\int_{0}^{t} \bar{h}(s, B(s)) d s-\frac{q-1}{2} \int_{\mathbb{R}^{d+1}}|h(\lambda, \xi)|^{2} \mu_{0}(d \lambda) \mu(d \xi)\right\},
\end{aligned}
$$

where $q=p(p-1)^{-1}$,

$$
\begin{aligned}
\mathcal{H}_{V}= & \left\{h \in \mathcal{L}^{2}\left(\mathbb{R}^{d+1}, \mu_{0} \otimes \mu\right) ; h\right. \text { is bounded and locally supported and } \\
& \left.h(-\lambda,-\xi)=\overline{h(\lambda, \xi)} \text { a.e. }-\mu_{0} \otimes \mu\right\}
\end{aligned}
$$

and, by the local finiteness and the symmetry of $\mu_{0}(d \lambda)$ and $\mu(d \xi)$, the function $\bar{h}(s, x)$ defined by

$$
\bar{h}(s, x) \equiv \int_{\mathbb{R}^{d+1}} \exp \{i(\lambda s+\xi \cdot x)\} h(\lambda, \xi) \mu_{0}(d \lambda) \mu(d \xi)
$$

is bounded, uniformly continuous on $[0,1] \times \mathbb{R}^{d}$ and real-valued. 
Proof. First, by stationarity of $u(t, x)$ in $x$, we may let $x=0$. We start by considering $u_{\varepsilon}(t, 0)$ instead of $u(t, 0)$. Recall that $\mathcal{L}^{q}(\Omega)$ stands for the space of all $V$-measurable and $q$-square integrable random variables and notice that

$$
\left\{\mathbb{E} u_{\varepsilon}^{p}(t, 0)\right\}^{1 / p}=\sup \left\{\mathbb{E}\left[X u_{\varepsilon}(t, 0)\right] ; X \in \mathcal{L}^{q}(\Omega) \text { with }\|X\|_{q}=1\right\} .
$$

For any real function $\varphi(u, x)$ on $\mathbb{R}^{d+1}$ with

$$
\int_{\mathbb{R}^{d+1} \times \mathbb{R}^{d+1}} \gamma_{0}(u-v) \gamma(x-y) \varphi(u, x) \varphi(v, y) d u d v d x d y<\infty
$$

take

$$
Z_{\varphi}=\exp \left\{\langle V, \varphi\rangle-\frac{1}{2} \int_{\mathbb{R}^{d+1}}|\mathcal{F}(\varphi)(\lambda, \xi)|^{2} \mu_{0}(d \lambda) \mu(d \xi)\right\} .
$$

We have that

$$
\left\{\mathbb{E} u_{\varepsilon}(t, x)^{p}\right\}^{1 / p} \geq \mathbb{E}\left\{\frac{Z_{\varphi}}{\left\|Z_{\varphi}\right\|_{q}} u_{\varepsilon}(t, 0)\right\} .
$$

From (2.2) it is easy to see that

$$
\left\|Z_{\varphi}\right\|_{q}=\exp \left\{\frac{q-1}{2} \int_{\mathbb{R}^{d+1}}|\mathcal{F}(\varphi)(\lambda, \xi)|^{2} \mu_{0}(d \lambda) \mu(d \xi)\right\} .
$$

In addition, by $(2.3)$ (with $u_{0}(\cdot)=1$ ) and Fubini's theorem

$$
\begin{aligned}
\mathbb{E}\left\{Z_{\varphi} u_{\varepsilon}(t, 0)\right\}= & \mathbb{E}_{0} \otimes \mathbb{E} \exp \left\{\left\langle V, \psi_{t}+\varphi\right\rangle-\frac{1}{2} \int_{\mathbb{R}^{d+1}}\left|\mathcal{F}\left(\psi_{t}\right)(\lambda, \xi)\right|^{2} \mu_{0}(d \lambda) \mu(d \xi)\right. \\
& \left.-\frac{1}{2} \int_{\mathbb{R}^{d+1}}|\mathcal{F}(\varphi)(\lambda, \xi)|^{2} \mu_{0}(d \lambda) \mu(d \xi)\right\} \\
= & \mathbb{E}_{0} \exp \left\{\int_{\mathbb{R}^{d+1}} \mathcal{F}(\varphi)(\lambda, \xi) \overline{\mathcal{F}\left(\psi_{t}\right)(\lambda, \xi)} \mu_{0}(d \lambda) \mu(d \xi)\right\} .
\end{aligned}
$$

Summarizing our computation,

$$
\begin{aligned}
\left(\mathbb{E} u_{\varepsilon}^{p}(t, 0)\right)^{1 / p} \geq & \mathbb{E}_{0} \exp \left\{\int_{\mathbb{R}^{d+1}} \mathcal{F}(\varphi)(\lambda, \xi) \overline{\mathcal{F}\left(\psi_{t}\right)(\lambda, \xi)} \mu_{0}(d \lambda) \mu(d \xi)\right. \\
& \left.-\frac{q-1}{2} \int_{\mathbb{R}^{d+1}}|\mathcal{F}(\varphi)(\lambda, \xi)|^{2} \mu_{0}(d \lambda) \mu(d \xi)\right\} .
\end{aligned}
$$

Notice that

$$
\begin{aligned}
& \int_{\mathbb{R}^{d+1}} \mathcal{F}(\varphi)(\lambda, \xi) \overline{\mathcal{F}\left(\psi_{t}\right)(\lambda, \xi)} \mu_{0}(d \lambda) \mu(d \xi) \\
& \quad=\int_{0}^{t}\left[\int_{\mathbb{R}^{d+1}} \mathcal{F}(\varphi)(\lambda, \xi) \exp \left\{-\frac{\varepsilon^{2}}{2}\left(\lambda^{2}+|\xi|^{2}\right)\right\} \exp \{i(\lambda(t-s)+\xi \cdot B(s))\} \mu_{0}(d \lambda) \mu(d \xi)\right] d s .
\end{aligned}
$$

Letting $\varepsilon \rightarrow 0^{+}$on the both sides of the inequality,

$$
\begin{aligned}
\left(\mathbb{E} u^{p}(t, 0)\right)^{1 / p} \geq & \mathbb{E}_{0} \exp \left\{\int_{0}^{t}\left[\int_{\mathbb{R}^{d+1}} \mathcal{F}(\varphi)(\lambda, \xi) \exp \{i(\lambda(t-s)+\xi \cdot B(s))\} \mu_{0}(d \lambda) \mu(d \xi)\right] d s\right. \\
& \left.-\frac{q-1}{2} \int_{\mathbb{R}^{d+1}}|\mathcal{F}(\varphi)(\lambda, \xi)|^{2} \mu_{0}(d \lambda) \mu(d \xi)\right\} .
\end{aligned}
$$


Finally, the claim follows from the fact that the linear map

$$
\varphi(u, x) \mapsto h(\lambda, \xi)=e^{-i \lambda t} \mathcal{F}(\varphi)(-\lambda, \xi)
$$

is an isometry between the Hilbert space

$$
\overline{\mathcal{H}}_{V}=\left\{h \in \mathcal{L}^{2}\left(\mathbb{R}^{d+1}, \mu_{0} \otimes \mu\right) ; h(-\lambda,-\xi)=\overline{h(\lambda, \xi)} \text { a.e. }-\mu_{0} \otimes \mu\right\}
$$

and the Hilbert space of the functions $\varphi$ on $\mathbb{R}^{d+1}$ that satisfy (2.5).

\section{Proof of the lower bounds}

Recall our assumption $u_{0}(\cdot)=1$. By the fact that $\mathbb{E} u(t, x)=1$ we need only to consider the case $n \geq 2$. Notice that

$$
\mu_{0}(d \lambda)=c_{0}|\lambda|^{-\left(1-\alpha_{0}\right)} d \lambda
$$

for some constant $c_{0}>0$. Consequently, $\mu_{0}(d(c \lambda))=c^{\alpha_{0}} \mu_{0}(d \lambda)$ for any constant $C>0$. Similarly (1.7), $\mu(d(c \xi))=$ $c^{\alpha} \mu(d \xi)$. Set

$$
t_{n}=t^{\frac{4-\alpha-2 \alpha_{0}}{2-\alpha}}(n-1)^{\frac{2}{2-\alpha}}=t^{\beta}(n-1)^{\frac{2}{2-\alpha}}, \quad \text { where } \beta=\frac{4-\alpha-2 \alpha_{0}}{2-\alpha} .
$$

We adopt the notations used in Proposition 2.1. For any symmetric function $h \in \mathcal{H}_{V}$, set

$$
h_{t}(\lambda, \xi)=t(n-1) h\left(t \lambda,(n-1)^{-\frac{1}{2-\alpha}} t^{\left.-\frac{\beta-1}{2} \xi\right)}\right. \text {. }
$$

By a linear integration substitution and by the Brownian scaling one can show that

$$
\int_{0}^{t_{n}} \bar{h}\left(\frac{s}{t_{n}}, B(s)\right) d s \stackrel{d}{=} \int_{0}^{t} \bar{h}_{t}(s, B(s)) d s
$$

and

$$
\int_{\mathbb{R}^{d+1}}\left|h_{t}(\lambda, \xi)\right|^{2} \mu_{0}(d \lambda) \mu(d \xi)=(n-1) t_{n} \int_{\mathbb{R}^{d+1}}|h(\lambda, \xi)|^{2} \mu_{0}(d \lambda) \mu(d \xi)
$$

By Proposition 2.1 (with $p=n$ )

$$
\begin{aligned}
& \left\{\mathbb{E} u^{n}(t, x)\right\}^{1 / n} \\
& \quad \geq \mathbb{E}_{0} \exp \left\{\int_{0}^{t} \bar{h}_{t}(s, B(s)) d s-\frac{1}{2(n-1)} \int_{\mathbb{R}^{d+1}}\left|h_{t}(\lambda, \xi)\right|^{2} \mu_{0}(d \lambda) \mu(d \xi)\right\} \\
& \quad=\mathbb{E}_{0} \exp \left\{\int_{0}^{t_{n}} \bar{h}\left(\frac{s}{t_{n}}, B(s)\right) d s-\frac{t_{n}}{2} \int_{\mathbb{R}^{d+1}}|h(\lambda, \xi)|^{2} \mu_{0}(d \lambda) \mu(d \xi)\right\} .
\end{aligned}
$$

According to Proposition 3.1, [5] and with the boundedness and the uniform continuity of $\bar{h}(s, x)$ on $[0,1] \times \mathbb{R}^{d}$ in mind,

$$
\begin{aligned}
& \lim _{t \rightarrow \infty} \frac{1}{t_{n}} \log \mathbb{E}_{0} \exp \left\{\int_{0}^{t_{n}} \bar{h}\left(\frac{s}{t_{n}}, B(s)\right) d s\right\} \\
& =\sup _{g \in \mathcal{A}_{d}}\left\{\int_{0}^{1} \int_{\mathbb{R}^{d}} \bar{h}(s, x) g^{2}(s, x) d x d s-\frac{1}{2} \int_{0}^{1} \int_{\mathbb{R}^{d}}\left|\nabla_{x} g(s, x)\right|^{2} d x d s\right\} .
\end{aligned}
$$


Notice that

$$
\int_{0}^{1} \int_{\mathbb{R}^{d}} \bar{h}(s, x) g^{2}(s, x) d x d s=\int_{\mathbb{R}^{d+1}} h(\lambda, \xi) \mathcal{F}\left(g^{2}\right)(\lambda, \xi) \mu_{0}(d \lambda) \mu(d \xi) .
$$

So we have

$$
\begin{aligned}
& \liminf _{t \rightarrow \infty} t^{-\beta} \log \mathbb{E} u^{n}(t, x) \\
& \geq n(n-1)^{\frac{2}{2-\alpha}} \sup _{g \in \mathcal{A}_{d}}\left\{\Psi(h, g)-\frac{1}{2} \int_{0}^{1} \int_{\mathbb{R}^{d}}\left|\nabla_{x} g(s, x)\right|^{2} d x d s\right\},
\end{aligned}
$$

where

$$
\Psi(h, g)=\int_{\mathbb{R}^{d+1}} h(\lambda, \xi) \mathcal{F}\left(g^{2}\right)(\lambda, \xi) \mu_{0}(d \lambda) \mu(d \xi)-\frac{1}{2} \int_{\mathbb{R}^{d+1}}|h(\lambda, \xi)|^{2} \mu_{0}(d \lambda) \mu(d \xi) .
$$

Taking supremum over $h \in \mathcal{H}_{V}$ on the right-hand side

$$
\begin{aligned}
& \liminf _{t \rightarrow \infty} t^{-\beta} \log \mathbb{E} u^{n}(t, x) \\
& \geq n(n-1)^{\frac{2}{2-\alpha}} \sup _{g \in \mathcal{A}_{d}}\left\{\sup _{h \in \mathcal{H}_{V}} \Psi(h, g)-\frac{1}{2} \int_{0}^{1} \int_{\mathbb{R}^{d}}\left|\nabla_{x} g(s, x)\right|^{2} d x d s\right\} .
\end{aligned}
$$

Notice that $\mathcal{F}\left(g^{2}\right) \in \overline{\mathcal{H}}_{V}$ where $\overline{\mathcal{H}}_{V}$ is defined in (2.6). The functional $\Psi(h, g)$ is continuous in $h$ with respect to the $\mathcal{L}^{2}\left(\mathbb{R}^{d+1}, \mu_{0} \otimes \mu\right)$-norm. By the fact that $\overline{\mathcal{H}}_{V}$ is the closure of $\mathcal{H}_{V}$ in $\mathcal{L}^{2}\left(\mathbb{R}^{d+1}, \mu_{0} \otimes \mu\right)$, therefore,

$$
\sup _{h \in \mathcal{H}_{V}} \Psi(h, g)=\sup _{h \in \overline{\mathcal{H}}_{V}} \Psi(h, g) \text {. }
$$

In particular, taking

$$
h(\lambda, \xi)=\mathcal{F}\left(g^{2}\right)(-\lambda,-\xi)=\overline{\mathcal{F}\left(g^{2}\right)(\lambda, \xi)}
$$

gives

$$
\begin{aligned}
& \liminf _{t \rightarrow \infty} t^{-\beta} \log \mathbb{E} u^{n}(t, x) \\
& \geq n(n-1)^{\frac{2}{2-\alpha}} \sup _{g \in \mathcal{A}_{d}}\left\{\frac{1}{2} \int_{\mathbb{R}^{d+1}}\left|\mathcal{F}\left(g^{2}\right)(\lambda, \xi)\right|^{2} \mu_{0}(d \lambda) \mu(d \xi)-\frac{1}{2} \int_{0}^{1} \int_{\mathbb{R}^{d}}\left|\nabla_{x} g(s, x)\right|^{2} d x d s\right\} \\
& =n(n-1)^{\frac{\alpha}{2-\alpha}} \sup _{g \in \mathcal{A}_{d}}\left\{\frac{1}{2} \int_{0}^{1} \int_{0}^{1} \int_{\mathbb{R}^{d} \times \mathbb{R}^{d}} \frac{\gamma(x-y)}{|s-r|^{\alpha_{0}}} g^{2}(s, x) g^{2}(r, y) d x d y d r d s\right. \\
& \left.\quad-\frac{1}{2} \int_{0}^{1} \int_{\mathbb{R}^{d}}\left|\nabla_{x} g(s, x)\right|^{2} d x d s\right\} \\
& =n\left(\frac{n-1}{2}\right)^{\frac{2}{2-\alpha}} \mathcal{E},
\end{aligned}
$$

where the last step follows from the substitution

$$
g(s, x) \mapsto\left(\frac{1}{2}\right)^{\frac{d}{2(2-\alpha)}} g\left(s,\left(\frac{1}{2}\right)^{\frac{1}{2-\alpha}} x\right) .
$$

By far, we have completed the proof of the lower bound for (1.14). Started from (3.1), the proof of the lower bound for (1.20) is similar. 


\section{Proof of the upper bounds}

The case when $n=1$ is automatic to Theorem 1.1 as $\mathbb{E} u(t, x)=1$ (recall that $u_{0}(\cdot)=1$ ). In the following $n \geq 2$. We begin with the moment representation ([7])

$$
\begin{aligned}
\mathbb{E} u(t, x)^{n} & =\mathbb{E}_{0} \exp \left\{\frac{1}{2} \sum_{j \neq k}^{n} \int_{0}^{t} \int_{0}^{t} \frac{\gamma\left(B_{j}(s)-B_{k}(r)\right)}{|s-r|^{\alpha_{0}}} d r d s\right\} \\
& =\mathbb{E}_{0} \exp \left\{\frac{1}{2(n-1)} \frac{1}{t_{n}} \sum_{j \neq k}^{n} \int_{0}^{t_{n}} \int_{0}^{t_{n}} \frac{\gamma\left(B_{j}(s)-B_{k}(r)\right)}{\left|t_{n}^{-1}(s-r)\right|^{\alpha_{0}}} d r d s\right\},
\end{aligned}
$$

where $B_{1}(s), \ldots, B_{n}(s)$ are independent $d$-dimensional Brownian motions,

$$
t_{n}=t^{\frac{4-\alpha-2 \alpha_{0}}{2-\alpha}}(n-1)^{\frac{2}{2-\alpha}}
$$

and the second step follows from Brownian scaling and time-space homogeneity of the covariance functions.

By variable substitution, the upper bounds for (1.14) and for (1.20) are reduced to the proof of

$$
\limsup _{t \rightarrow \infty} \frac{1}{t} \log \mathbb{E}_{0} \exp \left\{\frac{1}{2(n-1) t} \sum_{j \neq k}^{n} \int_{0}^{t} \int_{0}^{t} \frac{\gamma\left(B_{j}(s)-B_{k}(r)\right)}{\left|t^{-1}(s-r)\right|^{\alpha_{0}}} d r d s\right\} \leq n\left(\frac{1}{2}\right)^{\frac{2}{2-\alpha}} \mathcal{E}
$$

and

$$
\limsup _{n \rightarrow \infty} \frac{1}{n t_{n}} \log \mathbb{E}_{0} \exp \left\{\frac{1}{2(n-1) t_{n}} \sum_{j \neq k}^{n} \int_{0}^{t_{n}} \int_{0}^{t_{n}} \frac{\gamma\left(B_{j}(s)-B_{k}(r)\right)}{\left|t_{n}^{-1}(s-r)\right|^{\alpha_{0}}} d r d s\right\} \leq\left(\frac{1}{2}\right)^{\frac{2}{2-\alpha}} \mathcal{E},
$$

respectively.

\subsection{Reduction by exponential approximation}

To prove (4.1) and (4.2), the covariance functions have to be truncated properly. In this sub-section we legalize this procedure by approximation. First notice that

$$
|u|^{-\alpha_{0}}=c_{0}^{2} \int_{\mathbb{R}}|v-u|^{-\frac{1+\alpha_{0}}{2}}|v|^{-\frac{1+\alpha_{0}}{2}} d v \quad u \in \mathbb{R}
$$

for some constant $c_{0}>0$. Since the function $c_{0}|\cdot|^{-\frac{1+\alpha_{0}}{2}}$ can be approximated by a monotonic sequence of bounded, locally supported and continuous functions, for given $\delta>0$, there is a bounded, locally supported and continuous function $\psi(u)$ on $\mathbb{R}$ such that

$$
0 \leq \psi(u) \leq c_{0}|u|^{-\frac{1+\alpha_{0}}{2}} \quad \text { and } \quad \int_{0}^{1} \gamma_{0}(s) d s-\int_{0}^{1}\left[\int_{\mathbb{R}} \psi(v-s) \psi(v) d v\right] d s \leq \delta .
$$

Here we remark that that such approximation does not exist if $|\cdot|^{-\alpha_{0}}$ is replaced by $\delta_{0}(\cdot)$. That is why our argument fails in the setting when $V(t, x)$ is white in time.

Set

$$
\bar{\gamma}_{0}(u)=\int_{\mathbb{R}} \psi(v-u) \psi(v) d v, \quad \Delta \gamma_{0}(u)=|u|^{-\alpha_{0}}-\bar{\gamma}_{0}(u) \quad u \in \mathbb{R}
$$


and consider the decomposition

$$
\begin{aligned}
& \int_{0}^{t} \int_{0}^{t} \frac{\gamma\left(B_{j}(s)-B_{k}(r)\right)}{\left|t^{-1}(s-r)\right|^{\alpha_{0}}} d r d s \\
& =\int_{0}^{t} \int_{0}^{t} \bar{\gamma}_{0}\left(t^{-1}(s-r)\right) \gamma\left(B_{j}(s)-B_{k}(r)\right) d r d s \\
& \quad+\int_{0}^{t} \int_{0}^{t} \Delta \gamma_{0}\left(t^{-1}(s-r)\right) \gamma\left(B_{j}(s)-B_{k}(r)\right) d r d s .
\end{aligned}
$$

By Hölder inequality

$$
\begin{aligned}
\mathbb{E}_{0} \exp \left\{\frac{1}{2(n-1) t} \sum_{j \neq k}^{n} \int_{0}^{t} \int_{0}^{t} \frac{\gamma\left(B_{j}(s)-B_{k}(r)\right)}{\left|t^{-1}(s-r)\right|^{\alpha_{0}}} d r d s\right\} \\
\leq\left(\mathbb{E}_{0} \exp \left\{\frac{p}{2(n-1) t} \sum_{j \neq k}^{n} \int_{0}^{t} \int_{0}^{t} \bar{\gamma}_{0}\left(t^{-1}(s-r)\right) \gamma\left(B_{j}(s)-B_{k}(r)\right) d r d s\right\}\right)^{1 / p} \\
\quad \times\left(\mathbb{E}_{0} \exp \left\{\frac{q}{2(n-1) t} \sum_{j \neq k}^{n} \int_{0}^{t} \int_{0}^{t} \Delta \gamma_{0}\left(t^{-1}(s-r)\right) \gamma\left(B_{j}(s)-B_{k}(r)\right) d r d s\right\}\right)^{1 / q},
\end{aligned}
$$

where $p, q>1$ are conjugate numbers with $p$ being close to 1 .

Despite that $q$ is large, in the following we show that the second factor on the right-hand side does not make any substantial contribution as $\delta>0$ in (4.4) is sufficiently small. A fact we use in our argument is that ([6]) under the Dalang's condition (1.5) (in particular, under (1.6))

$$
\mathbb{E}_{0} \exp \left\{\theta \int_{0}^{t} \gamma(B(s)) d s\right\}<\infty
$$

for any $\theta>0$ and $t>0$.

Notice that

$$
\begin{aligned}
\int_{0}^{t} & \int_{0}^{t} \Delta \gamma_{0}\left(t^{-1}(s-r)\right) \gamma\left(B_{j}(s)-B_{k}(r)\right) d r d s \\
= & \int_{0}^{t} \int_{0}^{s} \Delta \gamma_{0}\left(t^{-1}(s-r)\right) \gamma\left(B_{j}(s)-B_{k}(r)\right) d r d s \\
& \quad+\int_{0}^{t} \int_{0}^{s} \Delta \gamma_{0}\left(t^{-1}(s-r)\right) \gamma\left(B_{k}(r)-B_{j}(s)\right) d r d s \\
\leq & \int_{0}^{t} \Delta \gamma_{0}\left(t^{-1} s\right)\left[\int_{0}^{t} \gamma\left(B_{j}(s+r)-B_{k}(r)\right) d r\right] d s \\
& +\int_{0}^{t} \Delta \gamma_{0}\left(t^{-1} s\right)\left[\int_{0}^{t} \gamma\left(B_{k}(s+r)-B_{j}(r)\right) d r\right] d s .
\end{aligned}
$$

Consequently,

$$
\begin{aligned}
& \sum_{j \neq k}^{n} \int_{0}^{t} \int_{0}^{t} \Delta \gamma_{0}\left(t^{-1}(s-r)\right) \gamma\left(B_{j}(s)-B_{k}(r)\right) d r d s \\
& \quad \leq 2 \int_{0}^{t} \Delta \gamma_{0}\left(t^{-1} s\right)\left[\sum_{j \neq k}^{n} \int_{0}^{t} \gamma\left(B_{j}(s+r)-B_{k}(r)\right) d r\right] d s .
\end{aligned}
$$


By Jensen's inequality

$$
\begin{aligned}
& \mathbb{E}_{0} \exp \left\{\frac{q}{2(n-1) t} \sum_{j \neq k}^{n} \int_{0}^{t} \int_{0}^{t} \Delta \gamma_{0}\left(t^{-1}(s-r)\right) \gamma\left(B_{j}(s)-B_{k}(r)\right) d r d s\right\} \\
& \leq\left(\int_{0}^{t} \Delta \gamma_{0}\left(t^{-1} s\right) d s\right)^{-1} \int_{0}^{t} \Delta \gamma_{0}\left(t^{-1} s\right) \\
& \quad \times \mathbb{E}_{0} \exp \left\{\frac{q}{(n-1) t}\left(\int_{0}^{t} \Delta \gamma_{0}\left(t^{-1} s\right) d s\right) \sum_{j \neq k}^{n} \int_{0}^{t} \gamma\left(B_{j}(s+r)-B_{k}(r)\right) d r\right\} d s \\
& \leq\left(\int_{0}^{t} \Delta \gamma_{0}\left(t^{-1} s\right) d s\right)^{-1} \int_{0}^{t} \Delta \gamma_{0}\left(t^{-1} s\right) \mathbb{E}_{0} \exp \left\{\frac{q \delta}{n-1} \sum_{j \neq k}^{n} \int_{0}^{t} \gamma\left(B_{j}(s+r)-B_{k}(r)\right) d r\right\} d s,
\end{aligned}
$$

where the last step follows from (4.4).

Write

$$
\sum_{j \neq k}^{n} \int_{0}^{t} \gamma\left(B_{j}(s+r)-B_{k}(r)\right) d r=\sum_{j=1}^{n} \sum_{k: k \neq j}^{n} \gamma\left(B_{j}(s+r)-B_{k}(r)\right) d r .
$$

By Hölder's inequality again

$$
\begin{aligned}
& \mathbb{E}_{0} \exp \left\{\frac{q \delta}{n-1} \sum_{j \neq k}^{n} \int_{0}^{t} \gamma\left(B_{j}(s+r)-B_{k}(r)\right) d r\right\} \\
& \leq \prod_{j=1}^{n}\left(\mathbb{E}_{0} \exp \left\{\frac{n q \delta}{n-1} \sum_{k: k \neq j}^{n} \int_{0}^{t} \gamma\left(B_{j}(s+r)-B_{k}(r)\right) d r\right\}\right)^{\frac{1}{n}} \\
& =\mathbb{E}_{0} \exp \left\{\frac{n q \delta}{n-1} \sum_{k=2}^{n} \int_{0}^{t} \gamma\left(B_{1}(s+r)-B_{k}(r)\right) d r\right\}
\end{aligned}
$$

We now claim that for any $s>0$

$$
\begin{gathered}
\mathbb{E}_{0} \exp \left\{\frac{n q \delta}{n-1} \sum_{k=2}^{n} \int_{0}^{t} \gamma\left(B_{1}(s+r)-B_{k}(r)\right) d r\right\} \\
\leq\left(\mathbb{E}_{0} \exp \left\{\frac{n q \delta}{n-1} \int_{0}^{t} \gamma(B(r)) d r\right\}\right)^{n-1} .
\end{gathered}
$$

Write

$$
\sum_{k=2}^{n} \int_{0}^{t} \gamma\left(B_{1}(s+r)-B_{k}(r)\right) d r=\int_{0}^{t} \int_{\mathbb{R}^{d}} e^{i \xi \cdot B_{1}(s+r)} \sum_{k=2}^{n} e^{-i \xi \cdot B_{k}(r)} \mu(d \xi) d r
$$

and let $\widetilde{\mathbb{E}}_{0}$ be the expectation with respect to $\left\{B_{2}, \ldots, B_{n}\right\}$. Conditioning on $B_{1}$, for any integer $m \geq 1$

$$
\begin{aligned}
\widetilde{\mathbb{E}}_{0} & {\left[\sum_{k=2}^{n} \int_{0}^{t} \gamma\left(B_{1}(s+r)-B_{k}(r)\right) d r\right]^{m} } \\
& =\int_{[0, t]^{m}} \int_{\left(\mathbb{R}^{d}\right)^{m}} \exp \left\{i \sum_{l=1}^{m} \xi_{l} \cdot B_{1}\left(s+r_{l}\right)\right\} \mathbb{E}_{0}\left(\prod_{l=1}^{m} \sum_{k=2}^{n} e^{-i \xi_{l} \cdot B_{k}\left(r_{l}\right)}\right) \mu\left(d \xi_{1}\right) \cdots \mu\left(d \xi_{m}\right) d r_{1} \cdots d r_{m}
\end{aligned}
$$




$$
\begin{aligned}
& \leq \int_{[0, t]^{m}} \int_{\left(\mathbb{R}^{d}\right)^{m}} \mathbb{E}_{0}\left(\prod_{l=1}^{m} \sum_{k=2}^{n} e^{-i \xi_{l} \cdot B_{k}\left(r_{l}\right)}\right) \mu\left(d \xi_{1}\right) \cdots \mu\left(d \xi_{m}\right) d r_{1} \cdots d r_{m} \\
& =\mathbb{E}_{0}\left[\sum_{k=2}^{n} \int_{0}^{t} \gamma\left(B_{k}(r)\right) d r\right]^{m},
\end{aligned}
$$

where the inequality follows from the fact that

$$
\mathbb{E}_{0}\left(\prod_{l=1}^{m} \sum_{k=1}^{n} e^{-i \xi_{l} \cdot B_{k}\left(r_{l}\right)}\right)>0
$$

Thus, (4.7) follows from the Taylor expansion for exponential functions and the independence among $B_{2}, \ldots, B_{n}$.

Summarizing our computation since (4.6)

$$
\begin{aligned}
\mathbb{E}_{0} \exp \left\{\frac{q}{2(n-1) t} \sum_{j \neq k}^{n} \int_{0}^{t} \int_{0}^{t} \Delta \gamma_{0}\left(t^{-1}(s-r)\right) \gamma\left(B_{j}(s)-B_{k}(r)\right) d r d s\right\} \\
\leq\left(\mathbb{E}_{0} \exp \left\{\frac{n q \delta}{n-1} \int_{0}^{t} \gamma(B(r)) d r\right\}\right)^{n-1} .
\end{aligned}
$$

With an idea similar to (4.7), one can show that for any $t>0$

$$
\mathbb{E}_{x} \exp \left\{\frac{n q \delta}{n-1} \int_{0}^{t} \gamma(B(r)) d r\right\} \leq \mathbb{E}_{0} \exp \left\{\frac{n q \delta}{n-1} \int_{0}^{t} \gamma(B(r)) d r\right\}
$$

for any $x \in \mathbb{R}^{d}$. Combining this with Markov property

$$
\begin{aligned}
& \mathbb{E}_{0} \exp \left\{\frac{n q \delta}{n-1} \int_{0}^{t_{1}+t_{2}} \gamma(B(r)) d r\right\} \\
& \leq \mathbb{E}_{0} \exp \left\{\frac{n q \delta}{n-1} \int_{0}^{t_{1}} \gamma(B(r)) d r\right\} \mathbb{E}_{0} \exp \left\{\frac{n q \delta}{n-1} \int_{0}^{t_{2}} \gamma(B(r)) d r\right\}
\end{aligned}
$$

for any $t_{1}, t_{2}>0$. Consequently,

$$
\mathbb{E}_{0} \exp \left\{\frac{n q \delta}{n-1} \int_{0}^{t} \gamma(B(s)) d s\right\} \leq\left(\mathbb{E}_{0} \exp \left\{\frac{n q \delta}{n-1} \int_{0}^{1} \gamma(B(s)) d s\right\}\right)^{t}
$$

for any integer $t>1$. Thus

$$
\begin{aligned}
\mathbb{E}_{0} \exp \left\{\frac{q}{2(n-1) t} \sum_{j \neq k}^{n} \int_{0}^{t} \int_{0}^{t} \Delta \gamma_{0}\left(t^{-1}(s-r)\right) \gamma\left(B_{j}(s)-B_{k}(r)\right) d r d s\right\} \\
\leq\left(\mathbb{E}_{0} \exp \left\{\frac{n q \delta}{n-1} \int_{0}^{1} \gamma(B(s)) d s\right\}\right)^{(n-1) t} .
\end{aligned}
$$

Returning to (4.5), we have shown that for any $p>1$ (that can be arbitrarily close to 1), the second factor on the right-hand side can be made asymptotically negligible. Therefore, the proof of (4.1) is reduced to show that for any $\theta>0$

$$
\limsup _{t \rightarrow \infty} \frac{1}{t} \log \mathbb{E}_{0} \exp \left\{\frac{\theta}{2(n-1) t} \sum_{j \neq k}^{n} \int_{0}^{t} \int_{0}^{t} \bar{\gamma}_{0}\left(t^{-1}(s-r)\right) \gamma\left(B_{j}(s)-B_{k}(r)\right) d r d s\right\} \leq n\left(\frac{\theta}{2}\right)^{\frac{2}{2-\alpha}} \mathcal{E} .
$$


Replacing $t$ by $t_{n}$ in (4.5) and in almost same way, (4.2) is reduced to

$$
\begin{aligned}
& \limsup _{n \rightarrow \infty} \frac{1}{n t_{n}} \log \mathbb{E}_{0} \exp \left\{\frac{\theta}{2(n-1) t_{n}} \sum_{j \neq k}^{n} \int_{0}^{t_{n}} \int_{0}^{t_{n}} \bar{\gamma}_{0}\left(t_{n}^{-1}(s-r)\right) \gamma\left(B_{j}(s)-B_{k}(r)\right) d r d s\right\} \\
& \quad \leq\left(\frac{\theta}{2}\right)^{\frac{2}{2-\alpha}} \mathcal{E} .
\end{aligned}
$$

Recall that the function $K(\cdot)$ is given in (1.6) and notice that

$$
\begin{aligned}
& \sum_{j \neq k}^{n} \int_{0}^{t} \int_{0}^{t} \bar{\gamma}_{0}\left(t^{-1}(s-r)\right) \gamma\left(B_{j}(s)-B_{k}(r)\right) d r d s \\
& =\sum_{j, k=1}^{n} \int_{0}^{t} \int_{0}^{t} \bar{\gamma}_{0}\left(t^{-1}(s-r)\right) \gamma\left(B_{j}(s)-B_{k}(r)\right) d r d s \\
& \quad-\sum_{j=1}^{n} \int_{0}^{t} \int_{0}^{t} \bar{\gamma}_{0}\left(t^{-1}(s-r)\right) \gamma\left(B_{j}(s)-B_{j}(r)\right) d r d s \\
& =\int_{\mathbb{R}^{d+1}}\left[\sum_{j=1}^{n} \int_{0}^{t} \psi\left(u-t^{-1} s\right) K\left(x-B_{j}(s)\right) d s\right]^{2} d u d x \\
& \quad-\sum_{j=1}^{n} \int_{\mathbb{R}^{d+1}}\left[\int_{0}^{t} \psi\left(u-t^{-1} s\right) K\left(x-B_{j}(s)\right) d s\right]^{2} d u d x \\
& \leq(n-1) \sum_{j=1}^{n} \int_{\mathbb{R}^{d+1}}\left[\int_{0}^{t} \psi\left(u-t^{-1} s\right) K\left(x-B_{j}(s)\right) d s\right]^{2} d u d x
\end{aligned}
$$

where the last step follows from Jensen's inequality.

By independence,

$$
\begin{gathered}
\mathbb{E}_{0} \exp \left\{\frac{\theta}{2(n-1) t} \sum_{j \neq k}^{n} \int_{0}^{t} \int_{0}^{t} \bar{\gamma}_{0}\left(t^{-1}(s-r)\right) \gamma\left(B_{j}(s)-B_{k}(r)\right) d r d s\right\} \\
\leq\left(\mathbb{E}_{0} \exp \left\{\frac{\theta}{2 t} \int_{\mathbb{R}^{d+1}}\left[\int_{0}^{t} \psi\left(u-t^{-1} s\right) K(x-B(s)) d s\right]^{2} d u d x\right\}\right)^{n} .
\end{gathered}
$$

Therefore, both (4.8) and (4.9) have been further reduced to the same statement:

$$
\limsup _{t \rightarrow \infty} \frac{1}{t} \log \mathbb{E}_{0} \exp \left\{\frac{\theta}{2 t} \int_{\mathbb{R}^{d+1}}\left[\int_{0}^{t} \psi\left(u-t^{-1} s\right) K(x-B(s)) d s\right]^{2} d u d x\right\} \leq\left(\frac{\theta}{2}\right)^{\frac{2}{2-\alpha}} \mathcal{E}
$$

for any $\theta>0$.

Notice that (Section A.1, [2]) under (1.6), for any $C>0$ and $t>0$

$$
\mathbb{E}_{0} \exp \left\{C \int_{\mathbb{R}^{d}}\left[\int_{0}^{t} K(x-B(s)) d s\right]^{2} d x\right\}<\infty .
$$


Let $l: \mathbb{R}^{+} \longrightarrow[0,1]$ be a continuous function such that $l(u)=1$ on $[0,1]$ and $l(u)=0$ for on $[2, \infty)$. For each $\varepsilon>0$, define

$$
K_{\varepsilon}(x)=\left(p_{\varepsilon} * K\right)(x) l(\varepsilon|x|) \quad x \in \mathbb{R}^{d} .
$$

Clearly, $K_{\varepsilon}(\cdot)$ is continuous, locally supported, symmetric functions for each $\varepsilon>0$ and

$$
\lim _{\varepsilon \rightarrow 0^{+}} \mathbb{E}_{0} \exp \left\{C \int_{\mathbb{R}^{d}}\left[\int_{0}^{1}\left|K-K_{\varepsilon}\right|(x-B(s)) d s\right]^{2} d x\right\}=1 \quad C>0 .
$$

In addition, by Jensen's inequality

$$
\begin{aligned}
\int_{\mathbb{R}^{d+1}} & {\left[\int_{0}^{t} \psi\left(u-t^{-1} s\right) K(x-B(s)) d s\right]^{2} d u d x } \\
\leq & \frac{1}{b} \int_{\mathbb{R}^{d+1}}\left[\int_{0}^{t} \psi\left(u-t^{-1} s\right) K_{\varepsilon}(x-B(s)) d s\right]^{2} d u d x \\
& +\frac{1}{1-b} \int_{\mathbb{R}^{d+1}}\left[\int_{0}^{t} \psi\left(u-t^{-1} s\right)\left(K-K_{\varepsilon}\right)(x-B(s)) d s\right]^{2} d u d x
\end{aligned}
$$

for any $0<b<1$. Here we let $b$ be fixed but close to 1 . By the fact that $\psi(\cdot)$ is bounded and locally supported, there is a constant $C>0$ independent of $t$, such that

$$
\int_{\mathbb{R}^{d+1}}\left[\int_{0}^{t} \psi\left(u-t^{-1} s\right)\left(K-K_{\varepsilon}\right)(x-B(s)) d s\right]^{2} d u d x \leq C \int_{\mathbb{R}^{d}}\left[\int_{0}^{t}\left|K-K_{\varepsilon}\right|(x-B(s)) d s\right]^{2} d x .
$$

Combining this with Hölder's inequality,

$$
\begin{aligned}
\mathbb{E}_{0} \exp \left\{\frac{\theta}{2 t} \int_{\mathbb{R}^{d+1}}\left[\int_{0}^{t} \psi\left(u-t^{-1} s\right) K(x-B(s)) d s\right]^{2} d u d x\right\} \\
\leq\left(\mathbb{E}_{0} \exp \left\{\frac{\theta}{2 t} \frac{p}{b} \int_{\mathbb{R}^{d+1}}\left[\int_{0}^{t} \psi\left(u-t^{-1} s\right) K_{\varepsilon}(x-B(s)) d s\right]^{2} d u d x\right\}\right)^{1 / p} \\
\times\left(\mathbb{E}_{0} \exp \left\{\frac{\theta}{2 t} \frac{C q}{1-b} \int_{\mathbb{R}^{d}}\left[\int_{0}^{t}\left|K-K_{\varepsilon}\right|(x-B(s)) d s\right]^{2} d x\right\}\right)^{1 / q} .
\end{aligned}
$$

For any $t_{1}, t_{2}>0$, by Jensen's inequality

$$
\begin{aligned}
& \frac{1}{t_{1}+t_{2}} \int_{\mathbb{R}^{d}}\left[\int_{0}^{t_{1}+t_{2}}\left|K-K_{\varepsilon}\right|(x-B(s)) d s\right]^{2} d x \\
& \leq \frac{1}{t_{1}} \int_{\mathbb{R}^{d}}\left[\int_{0}^{t_{1}}\left|K-K_{\varepsilon}\right|(x-B(s)) d s\right]^{2} d x \\
& \quad+\frac{1}{t_{2}} \int_{\mathbb{R}^{d}}\left[\int_{t_{1}}^{t_{1}+t_{2}}\left|K-K_{\varepsilon}\right|(x-B(s)) d s\right]^{2} d x \\
& =\frac{1}{t_{1}} \int_{\mathbb{R}^{d}}\left[\int_{0}^{t_{1}}\left|K-K_{\varepsilon}\right|(x-B(s)) d s\right]^{2} d x \\
& \quad+\frac{1}{t_{2}} \int_{\mathbb{R}^{d}}\left[\int_{0}^{t_{2}}\left|K-K_{\varepsilon}\right|\left(x-B\left(t_{1}+s\right)-B\left(t_{1}\right)\right) d s\right]^{2} d x .
\end{aligned}
$$


By the increment independence,

$$
\begin{aligned}
\mathbb{E}_{0} \exp \left\{\frac{\theta}{2\left(t_{1}+t_{2}\right)} \frac{C q}{1-b} \int_{\mathbb{R}^{d}}\left[\int_{0}^{t_{1}+t_{2}}\left|K-K_{\varepsilon}\right|(x-B(s)) d s\right]^{2} d x\right\} \\
\leq \mathbb{E}_{0} \exp \left\{\frac{\theta}{2 t_{1}} \frac{C q}{1-b} \int_{\mathbb{R}^{d}}\left[\int_{0}^{t_{1}}\left|K-K_{\varepsilon}\right|(x-B(s)) d s\right]^{2} d x\right\} \\
\times \mathbb{E}_{0} \exp \left\{\frac{\theta}{2 t_{2}} \frac{C q}{1-b} \int_{\mathbb{R}^{d}}\left[\int_{0}^{t_{2}}\left|K-K_{\varepsilon}\right|(x-B(s)) d s\right]^{2} d x\right\} .
\end{aligned}
$$

Therefore, for integer $t>1$,

$$
\begin{aligned}
\mathbb{E}_{0} & \exp \left\{\frac{\theta}{2 t} \frac{C q}{1-b} \int_{\mathbb{R}^{d}}\left[\int_{0}^{t}\left|K-K_{\varepsilon}\right|(x-B(s)) d s\right]^{2} d x\right\} \\
\leq & \left(\mathbb{E}_{0} \exp \left\{\frac{\theta}{2} \frac{C q}{1-b} \int_{\mathbb{R}^{d}}\left[\int_{0}^{1}\left|K-K_{\varepsilon}\right|(x-B(s)) d s\right]^{2} d x\right\}\right)^{t} .
\end{aligned}
$$

Consequently,

$$
\begin{aligned}
& \limsup _{t \rightarrow \infty} \frac{1}{t} \log \mathbb{E}_{0} \exp \left\{\frac{\theta}{2 t} \frac{C q}{1-b} \int_{\mathbb{R}^{d}}\left[\int_{0}^{t}\left|K-K_{\varepsilon}\right|(x-B(s)) d s\right]^{2} d x\right\} \\
& \leq \log \mathbb{E}_{0} \exp \left\{\frac{\theta}{2} \frac{C q}{1-b} \int_{\mathbb{R}^{d}}\left[\int_{0}^{1}\left|K-K_{\varepsilon}\right|(x-B(s)) d s\right]^{2} d x\right\} .
\end{aligned}
$$

In view of (4.12), the right-hand side can be made arbitrarily small by letting $\varepsilon$ sufficiently small. Returning to (4.13) and noticing that $p$ and $b$ can be arbitrarily close to 1, the proof of (4.10), and therefore the proof of the upper bounds for Theorem 1.1 and Theorem 1.2, is finally reduced to the verification of the limiting bound:

$$
\limsup _{t \rightarrow \infty} \frac{1}{t} \log \mathbb{E}_{0} \exp \left\{\frac{\theta}{2 t} \int_{\mathbb{R}^{d+1}}\left[\int_{0}^{t} \psi\left(u-t^{-1} s\right) K_{\mathcal{E}}(x-B(s)) d s\right]^{2} d u d x\right\} \leq\left(\frac{\theta}{2}\right)^{\frac{2}{2-\alpha}} \mathcal{E}
$$

for every $\theta>0$ and $\varepsilon>0$.

\subsection{Proof of (4.14)}

Let $\varepsilon>0$ be fixed. Our first step is to build some sort of tightness for our model. Take $M>0$ sufficiently large so that the support sets of $\psi(\cdot)$ and $K_{\varepsilon}(\cdot)$ are contained in $[-M / 2, M / 2]$ and $[-M / 2, M / 2]^{d}$, respectively. Notice that

$$
\begin{aligned}
& \int_{\mathbb{R}^{d+1}}\left[\int_{0}^{t} \psi\left(u-t^{-1} s\right) K_{\varepsilon}(x-B(s)) d s\right]^{2} d u d x \\
& \quad=\sum_{k \in \mathbb{Z}} \sum_{z \in \mathbb{Z}^{d}} \int_{[-M, M]^{d+1}}\left[\int_{0}^{t} \psi\left(2 k M+u-t^{-1} s\right) K_{\varepsilon}(2 z M+x-B(s)) d s\right]^{2} d u d x \\
& \quad \leq \int_{[-M, M]^{d+1}}\left[\int_{0}^{t} \sum_{\left.k \in \mathbb{Z}_{z \in \mathbb{Z}^{d}} \sum \psi\left(2 k M+u-t^{-1} s\right) K_{\varepsilon}(2 z M+x-B(s)) d s\right]^{2} d u d x}\right. \\
& \quad=\int_{[-M, M]^{d+1}}\left[\int_{0}^{t} \widetilde{\psi}\left(u-t^{-1} s\right) \widetilde{K}_{\varepsilon}(x-B(s)) d s\right]^{2} d u d x
\end{aligned}
$$


where

$$
\widetilde{\psi}(u)=\sum_{k \in \mathbb{Z}} \psi(2 k M+u) \quad \text { and } \quad \widetilde{K}_{\varepsilon}(x)=\sum_{z \in \mathbb{Z}^{d}} K_{\varepsilon}(2 z M+x) .
$$

An observation critical to the proof is that $\widetilde{\psi}(u)$ and $\widetilde{K}_{\varepsilon}(x)$ are the periodic extensions of $\psi(u)$ and $K_{\varepsilon}(x)$, respectively. In particular, they are uniformly continuous and bounded (with the same supremum bounds as $\psi(u)$ and $K_{\varepsilon}(x)$, respectively). Consequently, for any integer $k \geq 1$ there is a $\delta_{k}>0$ such that

$$
\max \left\{\left|\widetilde{\psi}(u) \widetilde{K}_{\varepsilon}(x)-\widetilde{\psi}(v) \widetilde{K}_{\varepsilon}(y)\right| ;(u, v),(v, y) \in \mathbb{R}^{d+1} \text { and }|(u, x)-(v, y)| \leq \delta_{k}\right\} \leq \frac{1}{k} .
$$

Notice

$$
A \equiv \sup _{(u, x) \in \mathbb{R}^{d+1}} \psi(u) K_{\varepsilon}(x)<\infty
$$

and define the subset $\mathcal{C} \subset \mathcal{L}^{2}\left([-M, M]^{d+1}\right)$ as

$$
\begin{aligned}
\mathcal{C}= & \bigcap_{k=1}^{\infty}\left\{h \in \mathcal{L}^{2}\left([-M, M]^{d+1}\right) ; \sup _{|(u, x)-(v, y)| \leq \delta_{k}}|h(u, x)-h(v, y)| \leq \frac{1}{k}\right. \\
& \text { and } \left.0 \leq h(u, x) \leq A \forall(u, x) \in[-M, M]^{d+1}\right\} .
\end{aligned}
$$

The class $\mathcal{C}$ is uniformly bounded and equicontinuous. When viewed as the subset of $C\left([-M, M]^{d+1}\right), \mathcal{C}$ is relatively compact, according to Arzelá-Ascoli theorem. Consequently, $\mathcal{C}$ is also relatively compact in $\mathcal{L}^{2}\left([-M, M]^{d+1}\right)$ as the uniform convergence on $[-M, M]^{d+1}$ implies the $\left.\mathcal{L}^{2}\left([-M, M]^{d+1}\right]\right)$-convergence.

Write

$$
Z_{t}(u, x)=\frac{1}{t} \int_{0}^{t} \widetilde{\psi}\left(u-t^{-1} s\right) \widetilde{K}_{\varepsilon}(x-B(s)) d s
$$

and view $Z_{t}(\cdot, \cdot)$ as a stochastic process taking values in $\mathcal{L}^{2}\left([-M, M]^{d+1}\right)$. It is not hard to see that $Z_{t}(\cdot, \cdot) \in \mathcal{C}$. Let $\mathcal{K}$ be the closure of $\mathcal{C}$ in $\mathcal{L}^{2}\left([-M, M]^{d+1}\right)$.

Let $a>0$ be a small but fixed number and write

$$
\mathcal{O}_{f}=\left\{h \in \mathcal{L}^{2}\left([-M, M]^{d+1}\right) ;\|h\|^{2}<-\|f\|^{2}+2\langle f, h\rangle+a\right\} \quad f \in \mathcal{K} .
$$

Notice that $f \in \mathcal{O}_{f}$ for each $f \in \mathcal{K}$. So the above family forms an open cover of $\mathcal{K}$. Let $\mathcal{O}_{f_{1}}, \ldots, \mathcal{O}_{f_{m}}$ be a finite sub-cover. By the fact that $Z_{t} \in \mathcal{K}$,

$$
\left\|Z_{t}\right\|^{2}<\max _{1 \leq j \leq m}\left\{-\left\|f_{j}\right\|^{2}+2\left\langle f_{j}, Z_{t}\right\rangle\right\}+a .
$$

Thus,

$$
\begin{aligned}
& \mathbb{E}_{0} \exp \left\{\frac{\theta}{2 t} \int_{[-M, M]^{d+1}}\left[\int_{0}^{t} \widetilde{\psi}\left(u-t^{-1} s\right) \widetilde{K}_{\varepsilon}(x-B(s)) d s\right]^{2} d u d x\right\} \\
& \leq e^{a \theta t / 2} \sum_{j=1}^{m} \exp \left\{-\frac{\theta t}{2}\left\|f_{j}\right\|^{2}\right\} \mathbb{E}_{0} \exp \left\{\theta t\left\langle f_{j}, Z_{t}\right\rangle\right\} .
\end{aligned}
$$


Combining this with (4.15),

$$
\begin{aligned}
& \limsup _{t \rightarrow \infty} \frac{1}{t} \log \mathbb{E}_{0} \exp \left\{\frac{\theta}{2 t} \int_{\mathbb{R}^{d+1}}\left[\int_{0}^{t} \psi\left(u-t^{-1} s\right) K_{\mathcal{E}}(x-B(s)) d s\right]^{2} d u d x\right\} \\
& \leq \frac{a \theta}{2}+\max _{1 \leq j \leq m}\left\{-\frac{\theta}{2}\left\|f_{j}\right\|^{2}+\limsup _{t \rightarrow \infty} \frac{1}{t} \log \mathbb{E}_{0} \exp \left\{\theta t\left\langle f_{j}, Z_{t}\right\rangle\right\}\right\} .
\end{aligned}
$$

Observe that

$$
\left\langle f_{j}, Z_{t}\right\rangle=\frac{1}{t} \int_{[-M . M]^{d+1}} f_{j}(u, x)\left[\int_{0}^{t} \widetilde{\psi}\left(u-t^{-1} s\right) \widetilde{K}_{\varepsilon}(x-B(s)) d s\right] d u d x=\frac{1}{t} \int_{0}^{t} \bar{f}_{j}\left(\frac{s}{t}, B(s)\right) d s,
$$

where

$$
\bar{f}(s, x)=\int_{[-M . M]^{d+1}} \tilde{\psi}(u-s) \widetilde{K}_{\varepsilon}(y-x) f(u, y) d u d y .
$$

Applying Proposition 3.1, [5] we have

$$
\begin{aligned}
& \lim _{t \rightarrow \infty} \frac{1}{t} \log \mathbb{E}_{0} \exp \left\{\theta \int_{0}^{t} \bar{f}_{j}\left(\frac{s}{t}, B(s)\right) d s\right\} \\
& =\sup _{g \in \mathcal{A}_{d}}\left\{\theta \int_{0}^{1} \int_{\mathbb{R}^{d}} \bar{f}_{j}(s, x) g^{2}(s, x) d x d s-\frac{1}{2} \int_{0}^{1} \int_{\mathbb{R}^{d}}\left|\nabla_{x} g(s, x)\right|^{2} d x d s\right\} .
\end{aligned}
$$

By Fubini's theorem

$$
\begin{aligned}
\int_{0}^{1} \int_{\mathbb{R}^{d}} \bar{f}_{j}(s, x) g^{2}(s, x) d x d s & =\int_{[-M, M]^{d+1}} f_{j}(u, y)\left[\int_{0}^{1} \int_{\mathbb{R}^{d}} \widetilde{\psi}(u-s) \widetilde{K}_{\varepsilon}(y-x) g^{2}(s, x) d x d s\right] d u d y \\
& =\left\langle f_{j}, z(g)\right\rangle \quad \text { (say). }
\end{aligned}
$$

Summarizing our argument since (4.16)

$$
\begin{aligned}
& \limsup _{t \rightarrow \infty} \frac{1}{t} \log \mathbb{E}_{0} \exp \left\{\frac{\theta}{2 t} \int_{\mathbb{R}^{d+1}}\left[\int_{0}^{t} \psi\left(u-t^{-1} s\right) K_{\varepsilon}(x-B(s)) d s\right]^{2} d u d x\right\} \\
& \leq \frac{a \theta}{2}+\max _{1 \leq j \leq m} \sup _{g \in \mathcal{A}_{d}}\left\{-\frac{\theta}{2}\left\|f_{j}\right\|^{2}+\theta\left\langle f_{j}, z(g)\right\rangle-\frac{1}{2} \int_{0}^{1} \int_{\mathbb{R}^{d}}\left|\nabla_{x} g(s, x)\right|^{2} d x d s\right\} \\
& \quad \leq \frac{a \theta}{2}+\sup _{g \in \mathcal{A}_{d}}\left\{\frac{\theta}{2}\|z(g)\|^{2}-\frac{1}{2} \int_{0}^{1} \int_{\mathbb{R}^{d}}\left|\nabla_{x} g(s, x)\right|^{2} d x d s\right\},
\end{aligned}
$$

where the last step follows from the fact that $-\|f\|^{2}+2\langle f, h\rangle \leq\|h\|^{2}$ for $f, h \in \mathcal{L}^{2}\left([-M, M]^{d+1}\right)$. Letting $a \rightarrow 0^{+}$ on the right-hand side,

$$
\begin{aligned}
\limsup _{t \rightarrow \infty} & \frac{1}{t} \log \mathbb{E}_{0} \exp \left\{\frac{\theta}{2 t} \int_{\mathbb{R}^{d+1}}\left[\int_{0}^{t} \psi\left(u-t^{-1} s\right) K_{\varepsilon}(x-B(s)) d s\right]^{2} d u d x\right\} \\
\leq & \sup _{g \in \mathcal{A}_{d}}\left\{\frac{\theta}{2} \int_{[-M, M]^{d+1}}\left[\int_{0}^{1} \int_{\mathbb{R}^{d}} \widetilde{\psi}(u-s) \widetilde{K}_{\varepsilon}(y-x) g^{2}(s, x) d x d s\right]^{2} d u d y\right. \\
& \left.-\frac{1}{2} \int_{0}^{1} \int_{\mathbb{R}^{d}}\left|\nabla_{x} g(s, x)\right|^{2} d x d s\right\} .
\end{aligned}
$$


With a minor modification of Lemma A.3 in [5], the "lim $\sup _{M \rightarrow \infty}$ " of the variation on the right-hand side is no greater than

$$
\sup _{g \in \mathcal{A}_{d}}\left\{\frac{\theta}{2} \int_{\mathbb{R}^{d+1}}\left[\int_{0}^{1} \int_{\mathbb{R}^{d}} \psi(u-s) K_{\varepsilon}(y-x) g^{2}(s, x) d x d s\right]^{2} d u d y-\frac{1}{2} \int_{0}^{1} \int_{\mathbb{R}^{d}}\left|\nabla_{x} g(s, x)\right|^{2} d x d s\right\} .
$$

By our construction of $\psi(u)$ and $K_{\varepsilon}(x),(1.6)$ and (4.3)

$$
\begin{aligned}
& \int_{\mathbb{R}^{d+1}}\left[\int_{0}^{1} \int_{\mathbb{R}^{d}} \psi(u-s) K_{\varepsilon}(y-x) g^{2}(s, x) d x d s\right]^{2} d u d y \\
& \quad \leq \int_{\mathbb{R}^{d+1}}\left[\int_{0}^{1} \int_{\mathbb{R}^{d}} c_{0}|u-s|^{-\frac{1+\alpha_{0}}{2}}\left(p_{\varepsilon} * K\right)(y-x) g^{2}(s, x) d x d s\right]^{2} d u d y \\
& \leq \int_{\mathbb{R}^{d}} p_{\varepsilon}(z)\left\{\int_{\mathbb{R}^{d+1}}\left[\int_{0}^{1} \int_{\mathbb{R}^{d}} c_{0}|u-s|^{-\frac{1+\alpha_{0}}{2}} K(y-x-z) g^{2}(s, x) d x d s\right]^{2} d u d y\right\} d z \\
& =\int_{\mathbb{R}^{d+1}}\left[\int_{0}^{1} \int_{\mathbb{R}^{d}} c_{0}|u-s|^{-\frac{1+\alpha_{0}}{2}} K(y-x) g^{2}(s, x) d x d s\right]^{2} d u d y \\
& =\int_{0}^{1} \int_{0}^{1} \int_{\mathbb{R}^{d} \times \mathbb{R}^{d}} \frac{\gamma(x-y)}{|s-r|^{\alpha_{0}}} g^{2}(s, x) g^{2}(r, y) d x d y d s d r .
\end{aligned}
$$

In addition, under the substitution

$$
g(s, x) \mapsto\left(\frac{\theta}{2}\right)^{\frac{d}{2(2-\alpha)}} g\left(s,\left(\frac{\theta}{2}\right)^{\frac{1}{2-\alpha}} x\right)
$$

we have

$$
\sup _{g \in \mathcal{A}_{d}}\left\{\frac{\theta}{2} \int_{0}^{1} \int_{0}^{1} \int_{\mathbb{R}^{d} \times \mathbb{R}^{d}} \frac{\gamma(x-y)}{|s-r|^{\alpha_{0}}} g^{2}(s, x) g^{2}(r, y) d x d y d s d r-\frac{1}{2} \int_{0}^{1} \int_{\mathbb{R}^{d}}\left|\nabla_{x} g(s, x)\right|^{2} d x d s\right\}=\left(\frac{\theta}{2}\right)^{\frac{2}{2-\alpha}} \mathcal{E} .
$$

Combining the above variational estimate with (4.17), we have completed the proof of (4.14).

\section{Variational bounds}

In this section, we prove that the variations appearing in this work are finite under our assumptions. Further, for future reference, we consider a more general setting. In the following discussion, $\gamma_{0}(u)$ and $\gamma(x)$ are non-negative definite and can be some generalized functions defined only in the distributional sense. An example is when $V(t, x)=$ $\dot{W}^{\left(H_{0}, H\right)}(t, x)$ with $d=1$, the $(1+1)$-fractional noise with the Hurst parameters $1 / 2 \leq H_{0}<1$ and $0<H<1 / 2$. In this case the Dalang's condition is satisfied with

$$
\mu(d \xi)=C|\xi|^{1-2 H} d \xi .
$$

On the other hand, the condition " $\gamma(\cdot) \geq 0$ " implied by the second assumption (with $K(\cdot) \geq 0$ ) in (1.6) is nolonger satisfied.

Lemma 5.1. Under the Dalang' condition (1.5)

$$
\sup _{g \in \mathcal{F}_{d}}\left\{\theta \int_{\mathbb{R}^{d} \times \mathbb{R}^{d}} \gamma(x-y) g^{2}(x) g^{2}(y) d x d y-\frac{1}{2} \int_{\mathbb{R}^{d}}|\nabla g(x)|^{2} d x\right\}<\infty
$$

for any $\theta>0$, where $\mathcal{F}_{d}$ is the class defined in (1.16). 
Proof. Notice that

$$
\int_{\mathbb{R}^{d} \times \mathbb{R}^{d}} \gamma(x-y) g^{2}(x) g^{2}(y) d x d y=\int_{\mathbb{R}^{d}}\left|\mathcal{F}\left(g^{2}\right)(\xi)\right|^{2} \mu(d \xi),
$$

where

$$
\mathcal{F}\left(g^{2}\right)(\xi)=\int_{\mathbb{R}^{d}} e^{i \xi \cdot x} g^{2}(x) d x .
$$

By the fact that

$$
\mathcal{F}\left(g^{2}\right)(\xi)=\frac{i}{d} \int_{\mathbb{R}^{d}}\left(\frac{\xi}{|\xi|^{2}} \cdot \nabla g^{2}(x)\right) e^{i \xi \cdot x} d x
$$

we have the bound

$$
\begin{aligned}
\left|\mathcal{F}\left(g^{2}\right)(\xi)\right| & \leq \frac{1}{d}|\xi|^{-1} \int_{\mathbb{R}^{d}}\left|\nabla g^{2}(x)\right| d x \\
& =\frac{2}{d}|\xi|^{-1} \int_{\mathbb{R}^{d}}|g(x)||\nabla g(x)| d x \\
& \leq \frac{2}{d}|\xi|^{-1}\|g\|_{2} \cdot\|\nabla g\|_{2}=\frac{2}{d}|\xi|^{-1}\|\nabla g\|_{2} .
\end{aligned}
$$

In addition,

$$
\left|\mathcal{F}\left(g^{2}\right)(\xi)\right| \leq \mathcal{F}\left(g^{2}\right)(0)=\|g\|_{2}^{2}=1 .
$$

By the Dalang's condition (1.5), there is a $N>0$ such that

$$
\left(\frac{2}{d}\right)^{2} \int_{\{|\xi|>N\}}|\xi|^{-2} \mu(d \xi)<\frac{1}{2} \theta^{-1}
$$

Hence

$$
\begin{aligned}
\int_{\mathbb{R}^{d} \times \mathbb{R}^{d}} \gamma(x-y) g^{2}(x) g^{2}(y) d x d y & =\left\{\int_{\{|\xi| \leq N\}}+\int_{\{|\xi|>N\}}\right\}\left|\mathcal{F}\left(g^{2}\right)(\xi)\right|^{2} \mu(d \xi) \\
& \leq \mu(\{|\xi| \leq N\})+\left(\frac{2}{d}\right)^{2}\|\nabla g\|_{2}^{2} \int_{\{|\xi|>N\}}|\xi|^{-2} \mu(d \xi) \\
& \leq \mu(\{|\xi| \leq N\})+\frac{1}{2 \theta}\|\nabla g\|_{2}^{2}
\end{aligned}
$$

Therefore,

$$
\sup _{g \in \mathcal{F}_{d}}\left\{\theta \int_{\mathbb{R}^{d} \times \mathbb{R}^{d}} \gamma(x-y) g^{2}(x) g^{2}(y) d x d y-\frac{1}{2} \int_{\mathbb{R}^{d}}|\nabla g(x)|^{2} d x\right\} \leq \theta \mu(\{|\xi| \leq N\})<\infty .
$$

Lemma 5.2. Let $\gamma_{0}(u)(u \in \mathbb{R})$ and $\gamma(x)\left(x \in \mathbb{R}^{d}\right)$ be two non-negative definite functions such that $\gamma_{0}$ is locally integrable and $\gamma$ satisfies the Dalang's condition (1.5). Then

$$
\sup _{g \in \mathcal{A}_{d}}\left\{\theta \int_{0}^{1} \int_{0}^{1} \int_{\mathbb{R}^{d} \times \mathbb{R}^{d}} \gamma_{0}(s-r) \gamma(x-y) g^{2}(s, x) g^{2}(r, y) d x d y d r d s-\frac{1}{2} \int_{0}^{1} \int_{\mathbb{R}^{d}}\left|\nabla_{x} g(s, x)\right|^{2} x d s\right\}<\infty
$$

for any $\theta>0$. 
Proof. Let $g \in \mathcal{A}_{d}$ be fixed. Without changing notation, we extend $g(s, x)$ periodically (in $s$ ) from a function on $[0,1] \times \mathbb{R}^{d}$ to a function on $\mathbb{R}^{+} \times \mathbb{R}^{d}$

$$
\begin{aligned}
& \int_{0}^{1} \int_{0}^{1} \int_{\mathbb{R}^{d} \times \mathbb{R}^{d}} \gamma_{0}(r-s) \gamma(x-y) g^{2}(r, x) g^{2}(s, y) d x d y d r d s \\
& \quad=2 \int_{0}^{1} \int_{0}^{r} \int_{\mathbb{R}^{d} \times \mathbb{R}^{d}} \gamma_{0}(r-s) \gamma(x-y) g^{2}(r, x) g^{2}(s, y) d r d s \\
& \quad=2 \int_{0}^{1} \gamma_{0}(r)\left[\int_{0}^{1-r} \int_{\mathbb{R}^{d} \times \mathbb{R}^{d}} \gamma(x-y) g^{2}(s+r, x) g^{2}(s, y) d x d y d s\right] d r \\
& \quad \leq 2 \int_{0}^{1}\left|\gamma_{0}(r)\right|\left\{\int_{0}^{1}\left|\int_{\mathbb{R}^{d} \times \mathbb{R}^{d}} \gamma(x-y) g^{2}(s+r, x) g^{2}(s, y) d x d y\right| d s\right\} d r,
\end{aligned}
$$

where the second step follows from a variable substituion. Keep in mind that $\gamma_{0}(\cdot)$ and $\gamma(\cdot)$ are not necessarily nonnegative under the assumption of the lemma.

For fixed $0 \leq r, s \leq 1$,

$$
\begin{aligned}
\int_{\mathbb{R}^{d} \times \mathbb{R}^{d}} \gamma(x-y) g^{2}(s+r, x) g^{2}(s, y) d x d y \\
=\int_{\mathbb{R}^{d}}\left[\int_{\mathbb{R}^{d}} e^{i \xi \cdot x} g^{2}(s+r, x) d x\right] \overline{\left[\int_{\mathbb{R}^{d}} e^{i \xi \cdot x} g^{2}(s, x) d x\right]} \mu(d \xi) \\
\leq\left\{\int_{\mathbb{R}^{d}}\left|\int_{\mathbb{R}^{d}} e^{i \xi \cdot x} g^{2}(s+r, x) d x\right|^{2} \mu(d \xi)\right\}^{1 / 2}\left\{\int_{\mathbb{R}^{d}}\left|\int_{\mathbb{R}^{d}} e^{i \xi \cdot x} g^{2}(s, x) d x\right|^{2} \mu(d \xi)\right\}^{1 / 2} \\
=\left\{\int_{\mathbb{R}^{d} \times \mathbb{R}^{d}} \gamma(x-y) g^{2}(s+r, x) g^{2}(s+r, y) d x d y\right\}^{1 / 2} \\
\quad \times\left\{\int_{\mathbb{R}^{d} \times \mathbb{R}^{d}} \gamma(x-y) g^{2}(s, x) g^{2}(s, y) d x d y\right\}^{1 / 2} .
\end{aligned}
$$

Similarly,

$$
\begin{aligned}
& -\int_{\mathbb{R}^{d} \times \mathbb{R}^{d}} \gamma(x-y) g^{2}(s+r, x) g^{2}(s, y) d x d y \\
& \quad \leq\left\{\int_{\mathbb{R}^{d} \times \mathbb{R}^{d}} \gamma(x-y) g^{2}(s+r, x) g^{2}(s+r, y) d x d y\right\}^{1 / 2}\left\{\int_{\mathbb{R}^{d} \times \mathbb{R}^{d}} \gamma(x-y) g^{2}(s, x) g^{2}(s, y) d x d y\right\}^{1 / 2} .
\end{aligned}
$$

Consequently,

$$
\begin{aligned}
& \int_{0}^{1}\left|\int_{\mathbb{R}^{d} \times \mathbb{R}^{d}} \gamma(x-y) g^{2}(s+r, x) g^{2}(s, y) d x d y\right| d s \\
& \leq \int_{0}^{1}\left\{\int_{\mathbb{R}^{d} \times \mathbb{R}^{d}} \gamma(x-y) g^{2}(s+r, x) g^{2}(s+r, y) d x d y\right\}^{1 / 2} \\
& \quad \times\left\{\int_{\mathbb{R}^{d} \times \mathbb{R}^{d}} \gamma(x-y) g^{2}(s, x) g^{2}(s, y) d x d y\right\}^{1 / 2} d s \\
& \leq\left\{\int_{0}^{1} \int_{\mathbb{R}^{d} \times \mathbb{R}^{d}} \gamma(x-y) g^{2}(s+r, x) g^{2}(s+r, y) d x d y d s\right\}^{1 / 2}
\end{aligned}
$$




$$
\begin{aligned}
& \times\left\{\int_{0}^{1} \int_{\mathbb{R}^{d} \times \mathbb{R}^{d}} \gamma(x-y) g^{2}(s, x) g^{2}(s, y) d x d y d s\right\}^{1 / 2} \\
= & \int_{0}^{1} \int_{\mathbb{R}^{d} \times \mathbb{R}^{d}} \gamma(x-y) g^{2}(s, x) g^{2}(s, y) d x d y d s,
\end{aligned}
$$

where the last step follows from the time-periodicity.

Summarizing our computation since (5.1),

$$
\begin{aligned}
& \int_{0}^{1} \int_{0}^{1} \int_{\mathbb{R}^{d} \times \mathbb{R}^{d}} \gamma_{0}(r-s) \gamma(x-y) g^{2}(r, x) g^{2}(s, y) d x d y d r d s \\
& \quad \leq 2\left(\int_{0}^{1}\left|\gamma_{0}(r)\right| d r\right) \int_{0}^{1} \int_{\mathbb{R}^{d} \times \mathbb{R}^{d}} \gamma(x-y) g^{2}(s, x) g^{2}(s, y) d x d y d s .
\end{aligned}
$$

Thus,

$$
\begin{aligned}
& \sup _{g \in \mathcal{A}_{d}}\left\{\theta \int_{0}^{1} \int_{0}^{1} \int_{\mathbb{R}^{d} \times \mathbb{R}^{d}} \gamma_{0}(s-r) \gamma(x-y) g^{2}(s, x) g^{2}(r, y) d x d y d r d s-\frac{1}{2} \int_{0}^{1} \int_{\mathbb{R}^{d}}\left|\nabla_{x} g(s, x)\right|^{2} x d s\right\} \\
& \leq \sup _{g \in \mathcal{A}_{d}}\left\{2 \theta\left(\int_{0}^{1}\left|\gamma_{0}(r)\right| d r\right) \int_{0}^{1} \int_{\mathbb{R}^{d} \times \mathbb{R}^{d}} \gamma(x-y) g^{2}(s, x) g^{2}(s, y) d x d y d s-\frac{1}{2} \int_{0}^{1} \int_{\mathbb{R}^{d}}\left|\nabla_{x} g(s, x)\right|^{2} x d s\right\} \\
& \leq \int_{0}^{1} \sup _{g \in \mathcal{A}_{d}}\left\{2 \theta\left(\int_{0}^{1}\left|\gamma_{0}(r)\right| d r\right) \int_{\mathbb{R}^{d} \times \mathbb{R}^{d}} \gamma(x-y) g^{2}(s, x) g^{2}(s, y) d x d y-\frac{1}{2} \int_{\mathbb{R}^{d}}\left|\nabla_{x} g(s, x)\right|^{2} d x\right\} d s \\
& \quad=\sup _{g \in \mathcal{F}_{d}}\left\{2 \theta\left(\int_{0}^{1}\left|\gamma_{0}(r)\right| d r\right) \int_{\mathbb{R}^{d} \times \mathbb{R}^{d}} \gamma(x-y) g^{2}(x) g^{2}(y) d x d y-\frac{1}{2} \int_{\mathbb{R}^{d}}|\nabla g(x)|^{2} d x\right\} .
\end{aligned}
$$

The variation on the right-hand side is finite, according to Lemma 5.1.

\section{Acknowledgement}

The author is grateful to the anonymous referee(s) for careful reading of the manuscript and for making numerous corrections and suggestions.

\section{References}

[1] R. A. Carmona and S. A. Molchanov. Parabolic Anderson model and intermittency. Mem. Amer. Math. Soc. 108 (1994) 518. MR1185878

[2] X. Chen. Quenched asymptotics for Brownian motion in generalized Gaussian potential. Ann. Probab. 42 (2014) 576-622. MR3178468

[3] X. Chen. Precise intermittency for the parabolic Anderson equation with an $(1+1)$-dimensional time-space white noise. Ann. Inst. Henri Poincaré Probab. Stat. 51 (2015) 1486-1499. MR3414455

[4] X. Chen. Spatial asymptotics for the parabolic Anderson models with generalized time-space Gaussian noise. Ann. Probab. 44 (2016) 15351598. MR3474477

[5] X. Chen, Y. Z. Hu, J. Song and F. Xing. Exponential asymptotics for time-space Hamiltonians. Ann. Inst. Henri Poincaré Probab. Stat. 51 (2015) 1529-1561. MR3414457

[6] R. C. Dalang. Extending martingale measure stochastic integral with applications to spatially homogeneous S.P.D.E's. Electron. J. Probab. 4 (1999) 1-29. MR1684157

[7] Y. Z. Hu, J. Huang, D. Nualart and S. Tindel. Stochastic heat equations with general multiplicative Gaussian noise: Hölder continuity and intermittency. Electron. J. Probab. 20 (55) (2015) 1-50. MR3354615

[8] Y. Z. Hu and D. Nualart. Stochastic heat equation driven by fractional noise and local time. Probab. Theory Related Fields 143 (2009) 285-328. MR2449130

[9] Y. Z. Hu, D. Nualart and J. Song. Feynman-Kac formula for heat equation driven by fractional white noise. Ann. Probab. 39 (2011) $291-326$. MR2778803

[10] W. Hunziker and I. M. Sigal. The quantum N-body problem. J. Math. Phys. 41 (2000) 3448-3510. MR1768629

[11] M. Lewin, P. Nam and N. Rougerie. Derivation of Hartree's theory for generic mean-field Bose system. Adv. Math. 254 (2014) 570-621. MR3161107 